\title{
Survey of FY 2001 Nanotechnology Related Research at the National Institute of Standards and Technology
}

\author{
Edited by: \\ Chad R. Snyder \\ J. Patrick Looney \\ Michael P. Casassa \\ U.S. DEPARTMENT OF COMMERCE \\ Technology Administration \\ National Institute of Standards \\ and Technology \\ Gaithersburg, MD 20899
}

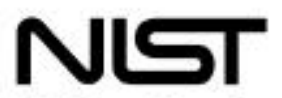

National Institute of Standards and Technology

Technology Administration

U.S. Department of Commerce 
NISTIR 6864

\section{Survey of FY 2001 Nanotechnology- Related Research at the National Institute of Standards and Technology}

Chad R. Snyder J. Patrick Looney Michael P. Casassa (editors)

Office of the Director

February 2002

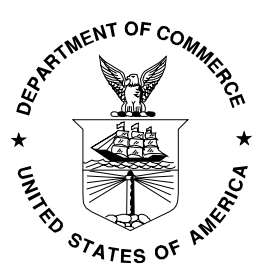

U.S. Department of Commerce Donald L. Evans, Secretary

Technology Administration Phillip J. Bond, Under Secretary for Technology

National Institute of Standards and Technology Arden L. Bement, Jr., Director 



\section{TABLE OF CONTENTS}

\section{INTRODUCTION}

The U.S. Government and Nanotechnology

NIST and Nanotechnology

Additional Remarks

\section{ELECTRONICS AND ELECTRICAL ENGINEERING LABORATORY (EEEL)}

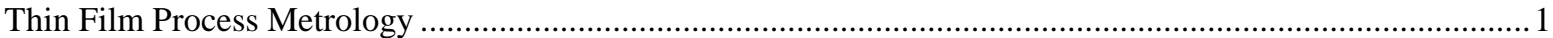

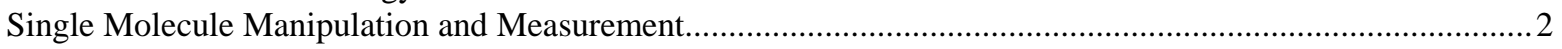

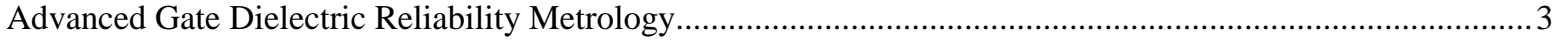

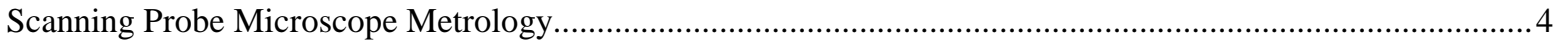

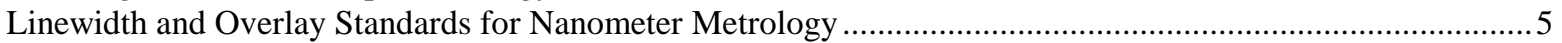

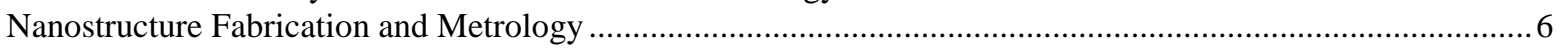

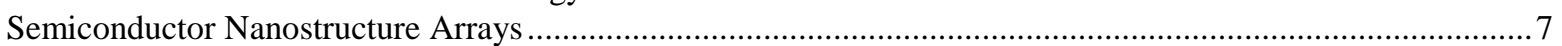

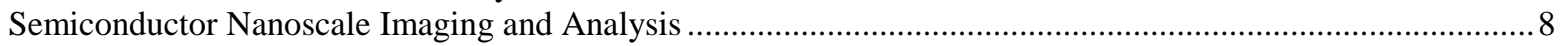

Nanoscopic Nonlinear Optical and Spectroscopic Metrologies in Wide-Bandgap Semiconductors .................9

Nanoscale Quantum Standards: Capacitance and Current Standards Based On Counting Electrons ................ 10

Nanoscale Quantum Standards: Arbitrary Waveform Generators for Standards, Communications, and Radar .11

Nanoscale Quantum Standards: Radiometry Standard Based on Counting Photons ..................................12

Nanoscale Quantum Standards: Quantum Time Standards and Computing (Fabrication)...........................13

X-ray Microanalysis of Nanometer Scale Particles and Films ......................................................... 14

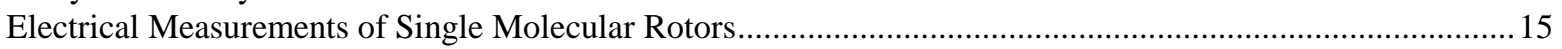

Rectification of Light as an Electrical Power Source ............................................................................ 16

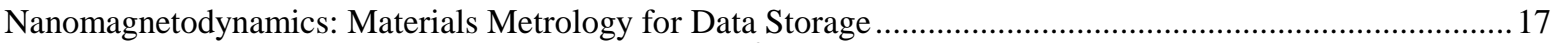

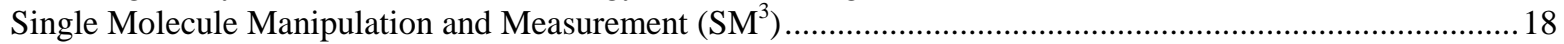

Coherent Spin Electronics for Microwave and Terahertz Communication and Signal Processing .................19

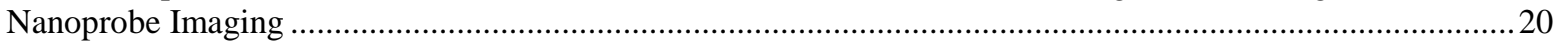

Emerging Applications of Nanoscale Magnetoresistive Sensors: Forensics, Non-destructive Failure Analysis,

Surveillance, Biological Monitoring....

\section{MANUFACTURING ENGINEERING LABORATORY (MEL)}

Atom-scale Length Measurement, Standards Fabrication, and Nanomanufacturing...................................22

Optical Manipulation for Testing and Assembly of Micro/Nanoscale Devices .....................................23

Scanning Electron Microscope (SEM) Length Metrology of Nanometer Structures .................................24

Optical Overlay and Linewidth Measurement Methods and Nanometer-scale Standards ...........................25

Scanned Probe Microscope Dimensional Metrology ......................................................................26

Fabrication and Characterization of Nanostructures for Standards..................................................27

Atomic-scale Motion Control for Nanometrology and Nanomanufacturing ...........................................28

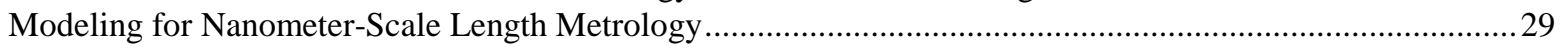

Microforce Realization and Measurement .............................................................................................30

\section{CHEMICAL SCIENCE AND TECHNOLOGY LABORATORY (CSTL)}

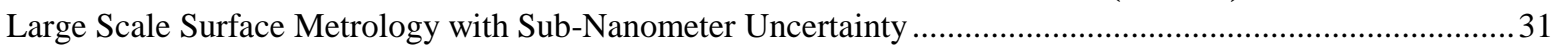

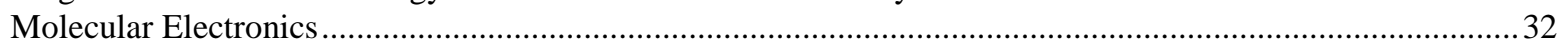

Self-Assembled Monolayers (SAMs) for Chemical and Biochemical Measurements .................................33

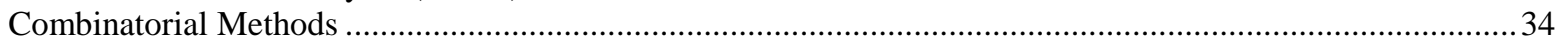

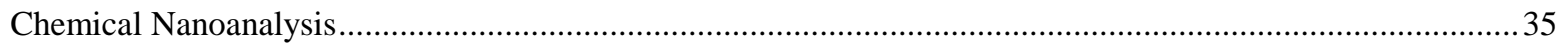

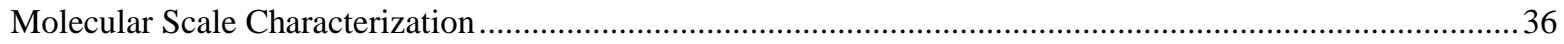

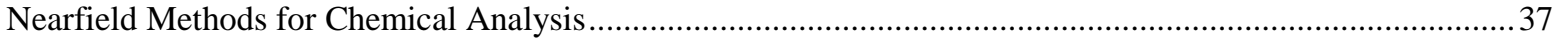


PHYSICS LABORATORY (PL)

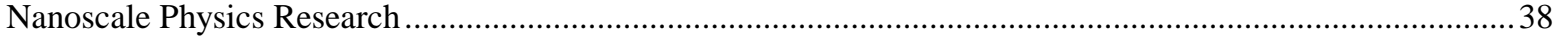

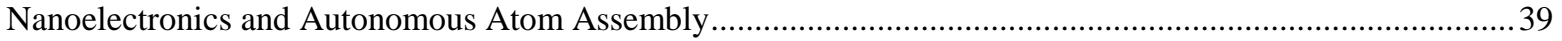

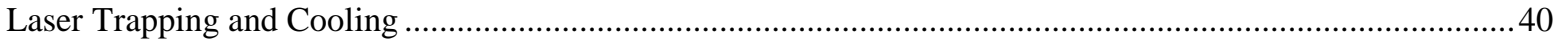

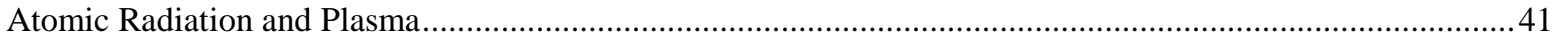

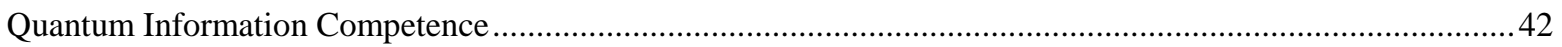

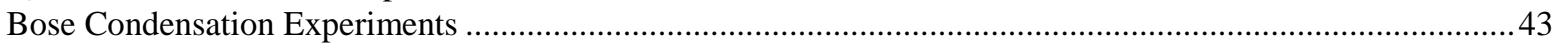

MATERIALS SCIENCE AND ENGINEERING LABORATORY (MSEL)

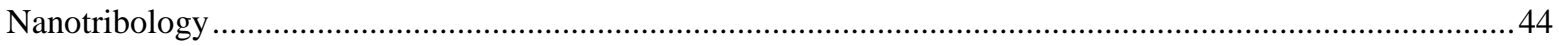

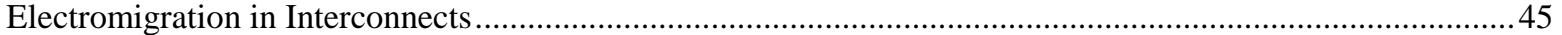

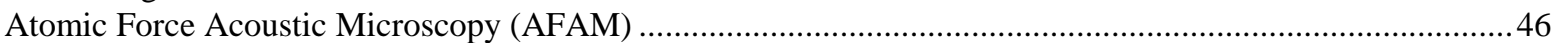

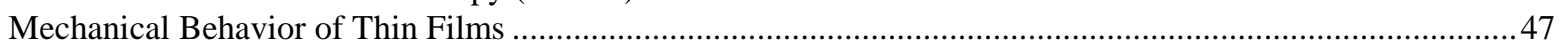

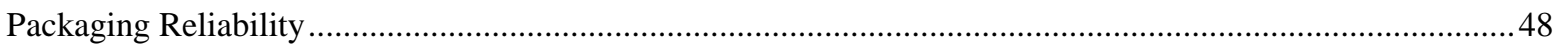

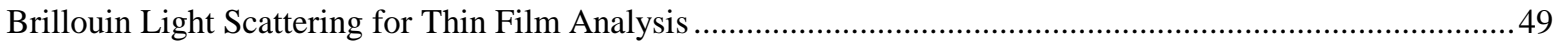

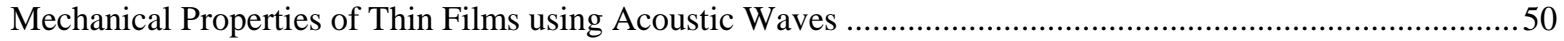

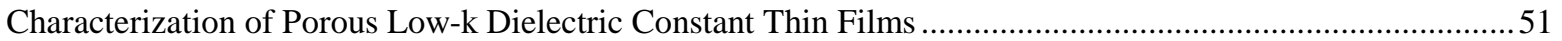

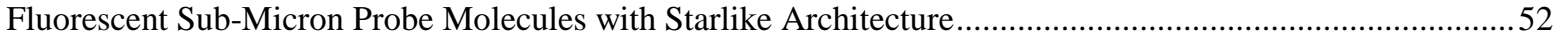

Fundamental Studies for the Development of Photoresists for Next-generation Photolithography: Thin Film

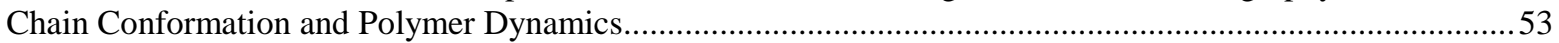

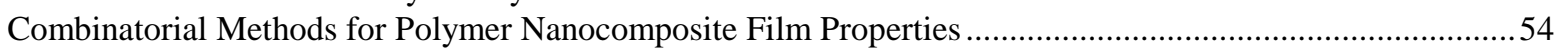

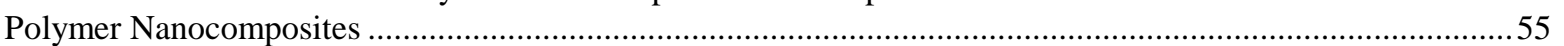

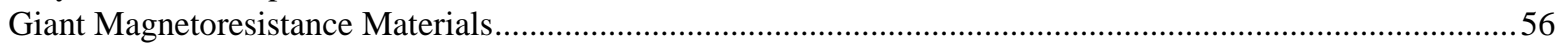

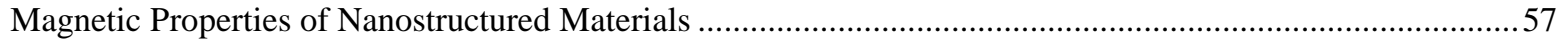

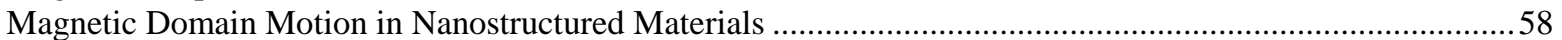

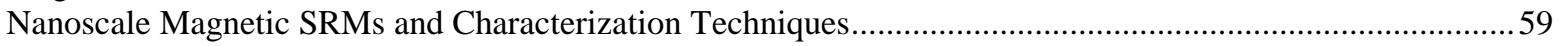

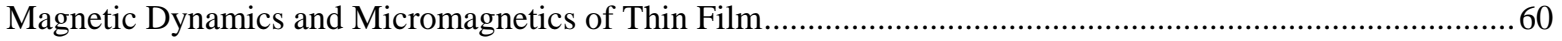

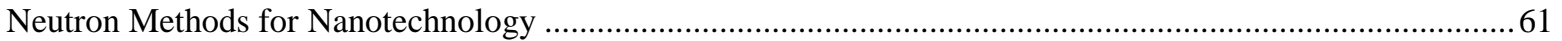

Neutron Studies of Nanoscale Films and Multilayers .................................................................6. 62

Neutron Scattering Investigations of Nanocomposites, Nanotubes and Self Assembled Structures ................63

\section{BUILDING AND FIRE RESEARCH LABORATORY (BFRL)}

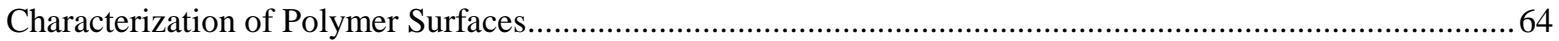

High-Performance Polymeric Building Materials: Polymer-Metal Oxide Nanocomposite Systems .................65

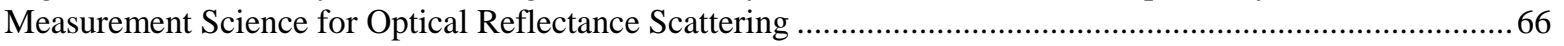

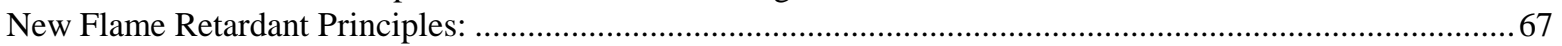

Particle Measurements in Support of the Semiconductor Industry ....................................................6.

A NIST Kinetic Data Base for PAH Reactions and Soot Particle Inception During Combustion ...................69

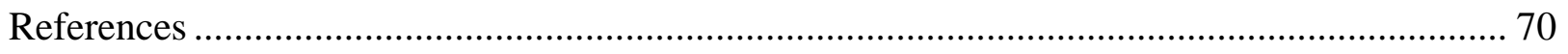




\section{INTRODUCTION}

\section{The U.S. Government and Nanotechnology}

Nanotechnology holds promise to dramatically change many aspects of the world in which we live. The range and scope of the potential economic and societal benefits from nanotechnologies is so staggering it has been called the "Next Industrial Revolution".[1] Recognizing the large potential for nanotechnologies, the FY2001 Federal Budget included support for a major new initiative on nanotechnology. The National Nanotechnology Initiative (NNI) was established by the U.S. Government to promote long-term nanoscale research and development leading to potential breakthroughs in areas ranging from materials and manufacturing to biotechnology and agriculture to national security and Defense, and many others. The NNI creates a research infrastructure by coordinating activities such as fundamental research, Grand Challenges (which will be described later), and centers and networks of excellence, activities that are all potentially high payoff and broadly enabling. The NNI evolved from publications authored by the Interagency Working Group on Nanoscience, Engineering and Technology (IWGN),[2] and is currently supported and monitored by the IWGN's successor, the Subcommittee on Nanoscale Science, Engineering, and Technology (NSET).[3] In FY 2001, the total investment by the NSET agencies in nanotechnology was estimated to be $\$ 422$ million, of which NIST has invested approximately $\$ 13.5$ million.

The NNI exemplifies the government's critical role in promoting the development of new science and technology. For clarity's sake, we should first consider what types of things we define as nanotechnology. Ask any scientist, engineer, or layperson for a definition of nanotechnology, and you will most likely receive a very different answer. In many cases, it is considered hard to define; like good art, most people "know it when they see it" but have difficulty explaining it in general. Thus, we use the definition of nanotechnology given by the NSET:

Nanotechnology is defined as the ability to work at the atomic, molecular or macromolecular levels, in the length scale of approximately $1-100 \mathrm{~nm}$ range, in order to create, manipulate and use structures, devices and systems that have novel properties and functions because of the small size. The novel and differentiating properties and functions are developed at a critical length scale of matter typically under $100 \mathrm{~nm}$. Nanotechnology includes integration of nanoscale structures into larger material components, systems and architectures that are used in most industries, health care systems, environment and national security. Within these larger scale devices, the control and construction of the devices remains at the nano scale. In some particular cases, the critical length scale for novel properties and phenomena may be under $1 \mathrm{~nm}$ (e.g., manipulation of atoms at $\sim 0.1 \mathrm{~nm}$ ) or be larger than $100 \mathrm{~nm}$ (e.g., nanoparticle reinforced polymers have the unique feature at 200-300 nm as a function of the local bridges or bonds between the nano particles and the polymer).[3]

It is critical that the Federal government is involved at this stage in the development of nanotechnology, since "the necessary fundamental nanotechnology research and development is too broad, complex, expensive, long-term, and risky for industry to undertake."[3] Industry is unable to fund or is under-funding critical areas of long-term fundamental research and 
development and is not developing the necessary nanoscience infratechnologies needed to realize nanotechnology's potential.

In supporting the NNI, the participating agencies fund the NNI recommended R\&D priorities as a function of their mission, contingent on available resources. The stated goals of the NSET involve developing a...

...coherent approach for funding the critical areas of nanoscience and engineering, establishing a balanced and flexible infrastructure, educating and training the necessary workforce, and promoting partnerships to ensure that these collective research activities provide a sound and balanced national research portfolio. By facilitating coordination and collaboration among agencies, the NNI will maximize the productivity and utility of the Federal government's investment in nanotechnology and avoid unnecessary duplication of efforts.[3]

\section{NIST and Nanotechnology}

This report catalogs the efforts of the National Institute of Standards and Technology (NIST) in nanotechnology during FY 2001. The purpose of this report is to provide a snapshot of current efforts in nanotechnology at NIST and in doing so allow one to view the role of NIST research in context of the total federal research nanotechnology effort and to understand NIST activities in the context of expected demands and strategic thrusts. The reason for NIST's broad interest in nanotechnology and participation in the NNI can be readily understood in terms of NIST's long standing presence in the measurements and standards arena and in NIST's role in promoting highrisk technology research in industry.

To ensure the commercial viability of nanotechnology-related products, nanoscale measurements and data will become critical to both process control and quality control. Additionally, measurements and data are also critical to developing a complete understanding of new phenomena. For these methods to succeed, several things need to be developed which can be grouped under the headings: metrology and infrastructure. For metrology, new approaches to analytical tools need to be developed and improvements must be made in the physical understanding of current instruments. Issues that arise in this category involve the uncertainty in interpreting measurements on molecular materials and the production of artifacts due to the measurement techniques themselves. Under the category of infrastructure, new measurements, standards, and data, are needed to bridge the gap between fundamental nanoscale science, engineering, and technology discoveries into new commercial products and services and to meet traceability requirements that may arise for international trade.

NIST's mission for measurements and standards for industry and other scientific interests goes back to its formation in 1901 by the Organic Act (Public Law 177-56 Congress). NIST 's Laboratories maintain the U.S. national standards for all fundamental measurements, including length, time, mass, electric current, temperature, etc., and for dozens of derived measurements. It is therefore not surprising that NIST should play a key role in enabling advances in nanoscale science, engineering, and technology at all levels. NIST is therefore taking the initiative to 
guarantee the accuracy and precision of nanoscale characterization equipment through scientific development (metrology), standards, and calibration sources.

NIST's role in nanotechnology is not limited to the research in its laboratories. NIST's Advanced Technology Program (ATP), has funded a series of nanotechnology-related projects in line with it mission to accelerate the development of innovative technologies for broad national benefit through partnerships with the private sector. The ATP co-funds high-risk research programs proposed and carried out by industry. These activities are cataloged in this report as well.

To place the NIST efforts in the context of the larger efforts of nanotechnologies within the US, we have added a matrix after each project/program listed in this report. The matrix is used to identify which of the "Grand Challenges" of nanotechnology, as defined by NSET $[3,4]$, that a particular project or program addresses.

Currently, the set of Grand Challenges of Nanotechnology, as defined by NSET, is:

\begin{tabular}{l|l} 
Grand Challenge & Symbol \\
\hline \hline nanostructured materials "by design" & MAT \\
nanoelectronics, optoelectronics and magnetics & ELEC \\
advanced healthcare, therapeutics and diagnostics & HEALTH \\
nanoscale processes for environmental improvement & ENV \\
efficient energy conversion and storage & ENER \\
microspacecraft exploration and industrialization & SPACE \\
bio-nanosensors for communicable disease and biological threat detection & BIOSEN \\
economical and safe transportation & TRANS \\
national security & SECUR \\
nanoscale experimental tools & MET \\
manufacturing at the nanoscale & MAN \\
\hline
\end{tabular}

Here the entries in the "Symbol" column are abbreviations used to help map NIST current programs onto the NSET Grand Challenges and are used in the matrix after each project/program, i.e.,

\begin{tabular}{|l|l|l|l|l|l|l|l|l|l|l|}
\hline MAT & ELEC & HEALTH & ENV & ENER & SPACE & BIOSEN & TRANS & SECUR & MET & MAN \\
\hline & & & & & & & & & & \\
\hline
\end{tabular}

By looking at the matrix after each of the program write-ups the relation of each of the NIST programs to the NSET Grand Challenges can be readily understood. 


\section{Additional Remarks}

The editors of this report assembled this survey, not to be comprehensive, but to give a flavor of the breadth of ongoing efforts at NIST in the nanotechnology arena. There are undoubtedly efforts at NIST that could be associated with nanotechnology, or will benefit nanotechnology in some way, which have not been listed in this report.

The editors would like to thank everyone who provided the information contained in this report and hope that the information contained within will be useful to the reader.

Chad R. Snyder

J. Patrick Looney

Michael P. Casassa

NIST Representatives to the NSET

February 2002 


\section{Electronics and Electrical Engineering Laboratory}

\section{Thin Film Process Metrology}

\section{Project Leader/contact:}

James Ehrstein, 301-975-2060, j.ehrstein@ nist.gov

Semiconductor Electronics Division, EEEL, Gaithersburg

Approximate staffing:

5 FTE

Funding sources

NIST (100\%)

Objective:

Develop improvements in optical and electrical measurements necessary for accurate characterization and process control of critical dielectric thin films for semiconductor technology, particular advanced gate dielectric films.

\section{Constituency:}

Semiconductor electronics industry

\section{Principal current tasks:}

- Develop improved film thickness metrology for the manufacturing environment

- Develop better understanding of the physics and functional models for optical-, electrical- and physicalthickness models

- Develop databases for the dielectric functions of advanced metal-oxide and metal-silicate films

- Develop means for rapid identification of process-induced changes in film composition or structure

- Develop mechanisms for the traceability of thin film measurements by industry to NIST.

Additional information:

These films are typically only several nanometers thick. The work focuses on developing; a) improved film thickness metrology for the manufacturing environment, b) better understanding of the physics and functional models for optical-, electrical- and physical- thickness models, c) databases for the dielectric functions of advanced metaloxide and metal-silicate films, and d) means for rapid identification of process-induced changes in film composition or structure. Finally, we seek to develop mechanisms for the traceability of thin film measurements by industry to NIST.

\section{Selected publications:}

1. Diebold, A., Canterbury, J., Chism, W., Richter, C. A., Nguyen, N. V., Ehrstein, J. R., and Weintraub, C., Characterization and Production Metrology of Gate Dielectric Films: Optical Models for Oxynitrides and High Dielectric Constant Films, Materials Science in Semiconductor Processing, vol. 4., pp. 3-8, 2001.

2. Richter, C. A., Nguyen, N. V., Gusev, E. P., Zabel, T. H., and Alers, G. B., Optical and Electrical Thickness Measurements of Alternate Gate Dielectrics: a Fundamental Difference, Characterization and Metrology for ULSI Technology: 2000, D. G. Seiler, A. C. Diebold, T. J. Shaffner, R. McDonald, W. M. Bullis, P. J. Smith, and E. M. Secula, Eds. (AIP, New York, 2001), pp. 134-139.

Alignment with NSET Grand Challenges:

\begin{tabular}{|c|c|c|l|l|l|l|l|c|c|c|}
\hline MAT & ELEC & HEALTH & ENV & ENER & SPACE & BIOSEN & TRANS & SECUR & MET & MAN \\
\hline $\mathrm{X}$ & $\mathrm{X}$ & & & & & & & & $\mathrm{X}$ & $\mathrm{X}$ \\
\hline
\end{tabular}




\section{Electronics and Electrical Engineering Laboratory}

\section{Single Molecule Manipulation and Measurement}

\section{Project Leader/contact:}

Michael Gaitan, 301-975-2070, michael.gaitan@ nist.gov

Semiconductor Electronics Division, EEEL, Gaithersburg

Approximate staffing:

3 FTE

Funding sources

NIST

\section{Objective:}

The objective is to develop single-molecule technologies to address significant inaccuracies in the base-pair ordering inferred by present gene-sequencing tools, affecting such well-publicized efforts as the Human Genome Project.

Constituency:

Biotechnology industries

Principal current tasks:

- Develop measurement methods for structural properties of deoxyribonucleic acid (DNA) using pores

- Develop the ability to interpret fluorescence resonance energy transfer (FRET) data unambiguously on single molecules and methods to measure the structure and dynamics of ribonucleic acid (RNA) standards and hairpin systems

- Develop the ability to measure single molecules spectroscopically, using surface enhanced Raman spectroscopy (SERS) and optically, using two-photon and UV excitation with simultaneous force measurements

- Develop fabrication methods for nanometer-scale elements and fluidic systems that will transport single molecules

Additional information:

This is part of a NIST-wide multi-Laboratory effort including CSTL, PL, and ITL in addition to EEEL. The objective is to develop single-molecule technologies to address significant inaccuracies in the base-pair ordering inferred by present gene-sequencing tools, affecting such well-publicized efforts as the Human Genome Project.

The program will ensure the integrity of such bio-informatic databases through the development and utilization of a high-throughput, single molecule manipulation and measurement $\left(\mathrm{SM}^{3}\right)$ platform to directly manipulate and measure the structure and dynamics of single RNA or DNA molecules. Within the five-year time span of this effort, the following will be developed: A platform will be produced that will combine these capabilities to make a single biomolecule measurement workbench.

Selected publications:

1. Herman, D., Gaitan, M., and DeVoe, D., MEMS Test Structures for Mechanical Characterization of VLSI Thin Films, Proc. SEM Conference, Portland, Oregon, Jun. 4-6, 2001, 5 p.

2. Rasmussen, A., Gaitan, M., Locascio, L., and Zaghloul, M., Fabrication Techniques to Realize CMOScompatible Microfluidic Microchannels, JMEMS, 10: (2) 286-297, June 2001.

Alignment with NSET Grand Challenges:

\begin{tabular}{|l|c|c|c|c|c|c|c|c|c|c|}
\hline MAT & ELEC & HEALTH & ENV & ENER & SPACE & BIOSEN & TRANS & SECUR & MET & MAN \\
\hline & & $\mathrm{X}$ & & & & & & & $\mathrm{X}$ & \\
\hline
\end{tabular}




\section{Electronics and Electrical Engineering Laboratory}

\section{Advanced Gate Dielectric Reliability Metrology}

\section{Project Leader/contact:}

John S. Suehle, 301-975-2247, john.suehle@ nist.gov

Semiconductor Electronics Division, EEEL, Gaithersburg

Approximate staffing:

3 FTE

Funding sources

NIST $(100 \%)$

\section{Objective:}

The objectives of this research are to develop measurement methods for determining the reliability of ultrathin gate dielectric materials, including $\mathrm{SiO}_{2}$ and candidate high-k materials. These measurement methods and the understanding developed about the reliability of gate dielectrics are transferred to the semiconductor industry through voluntary standards groups, collaborations, and publications.

Constituency:

Semiconductor electronics industry

Principal current tasks:

- Develop measurement methods for determining the reliability of ultrathin gate dielectric materials

\section{Additional information:}

Developing and manufacturing scaled, ultrathin gate dielectrics for future microelectronic devices is regarded as one of the most difficult challenges by the Semiconductor Industry Association (SIA) International Technology Roadmap for Semiconductors (ITRS). As the semiconductor industry scales device channel lengths to below $180 \mathrm{~nm}$, gate dielectrics must be scaled to have an equivalent oxide thickness below $2 \mathrm{~nm}$. Ultra-thin $\mathrm{SiO}_{2}$ (the gate dielectric that is used in present devices) exhibits large tunneling currents and the impact on device reliability is not well understood. An alternative, high-dielectric constant (high k) gate dielectric system will be required as devices are scaled further due to the excessive tunneling currents exhibited by sub- $2 \mathrm{~nm} \mathrm{SiO}_{2}$. The objectives of this research are to develop measurement methods for determining the reliability of ultrathin gate dielectric materials, including $\mathrm{SiO}_{2}$ and candidate high-k materials. These measurement methods and the understanding developed about the reliability of gate dielectrics are transferred to the semiconductor industry through voluntary standards groups, collaborations, and publications.

Selected publications:

1. Vogel, E. M., and Misra, V., MOS Device Characterization, in Handbook of Silicon Semiconductors Metrology, A. C. Diebold, Ed. (Marcel Dekker, Inc., New York - Basel, 2001), pp. 59-95.

2. Vogel, E. M., Edelstein, M. D., and Suehle, J. S., Defect Generation and Breakdown of Ultra-Thin Silicon Dioxide Induced by Substrate Hot Hole Injection, Journal of Applied Physics, vol. 90, no. 5, Sept. 1, 2001, pp. 2338-2347.

Alignment with NSET Grand Challenges:

\begin{tabular}{|c|c|c|c|c|c|c|c|c|c|c|}
\hline MAT & ELEC & HEALTH & ENV & ENER & SPACE & BIOSEN & TRANS & SECUR & MET & MAN \\
\hline & $X$ & & & & & & & & $X$ & \\
\hline
\end{tabular}




\section{Electronics and Electrical Engineering Laboratory}

\section{Scanning Probe Microscope Metrology}

\section{Project Leader/contact:}

Joseph Kopanski, 301-975-2089, joseph.kopanski@nist.gov

Semiconductor Electronics Division, EEEL, Gaithersburg

Approximate staffing:

4 FTE

Funding sources

NIST $(100 \%)$

Objective:

Provide silicon and compound semiconductor manufacturers with advanced scanning-probe electrical metrology techniques and models to improve device performance and reliability.

Constituency:

Semiconductor electronics industry

Principal current tasks:

- Provide the technology computer-aided design (TCAD) community with quantitative two-dimensional dopant profiles to calibrate and enhance the predictivity of process simulators

- Develop metrology techniques and data interpretation models for the scanning capacitance microscope (SCM)

\section{Additional information:}

Because scanning probe microscopes can make measurements with less than one nanometer spatial resolution, they are one of a very few techniques which can actually probe within the internal structure of state-of-the-art transistors. The goal of this project is to provide silicon and compound semiconductor manufacturers with advanced scanning-probe electrical metrology techniques and models to improve device performance and reliability.

Selected publications:

1. Marchiando, J. F., Kopanski, J. J., and Albers, J., Limitations of the Calibration Curve Method for Determining Dopant Profiles from Scanning Capacitance Microscope Measurements, J. Vac. Sci. Technol. B 18 (1), Jan/Feb 2000, pp. 414-417.

2. Kopanski, J. J., Marchiando, J. F., and Rennex, B. G., Scanning Capacitance Microscopy for Measuring Device Carrier Profiles Beyond the $100 \mathrm{~nm}$ Generation, Digest of Papers of the 2000 International Microprocesses and Nanotechnology Conference, at The University of Tokyo, Tokyo, Japan, July 11-13, 2000, pp. 250-251.

Alignment with NSET Grand Challenges:

\begin{tabular}{|c|c|c|c|c|c|c|c|c|c|c|}
\hline MAT & ELEC & HEALTH & ENV & ENER & SPACE & BIOSEN & TRANS & SECUR & MET & MAN \\
\hline & $\mathrm{X}$ & & & & & & & & $\mathrm{X}$ & \\
\hline
\end{tabular}




\section{Electronics and Electrical Engineering Laboratory}

\section{Linewidth and Overlay Standards for Nanometer Metrology}

Project Leader/contact

Michael W. Cresswell, 301-975-2072, michael.cresswell@ nist.gov

Semiconductor Electronics Division, EEEL, Gaithersburg

Approximate staffing:

5 FTE

Funding sources:

NIST $(100 \%)$

Objective:

Develop test-structure-based electrical metrology methods and related reference materials with primary emphasis on linewidth metrology and calibration; contribute to standards organizations supporting the development of metrology standards for the semiconductor tool industry.

Constituency:

Semiconductor electronics industry

Principal current tasks:

- Develop prototype critical dimension (CD) reference materials for calibrating linewidth metrology instruments

Additional information:

The technical approach developed for fabricating the prototype linewidth materials employs patterning with latticeplane selective etches of the kind used in silicon micromachining. This results in reference features with atomically planar sidewalls. Silicon wafers containing test chips with these features were then measured using low-cost, but very accurate and reproducible, electrical measurements. Since the accuracy of these electrical CD measurements was not known a priori, a selection of features were measured using High Resolution Transmission Electron Microscopy (HRTEM) to resolve the silicon lattice. HRTEM provides nanometer-level accuracy, but is sampledestructive and is prohibitively costly to implement for routine inspection. The lattice planes provide a built-in ruler (the separations of the silicon lattice planes are very accurately known) that is used to calibrate the electrical measurements. Typical reference features are several-hundred lattice planes wide. The prototype materials that have been delivered to ISMT have linewidths of $80 \mathrm{~nm}-140 \mathrm{~nm}$ and are intended for use with advanced circuits that will be introduced around the years 2003-2005.

Selected publications:

1. Cresswell, M. W., and Allen, R. A., Electrical CD Metrology and Related Reference Materials, in Handbook of Silicon Semiconductors Metrology, A. C. Diebold, Ed. (Marcel Dekker, Inc., New York - Basel, 2001), pp. 377-409.

2. Cresswell, M. W., Arora, N., Allen, R. A., Murabito, C. E., Richter, C. A., Gupta, A., Linholm, L. W., Pachura, D., and Bendix, P., Test Chip for Electrical Linewidth of Copper-Interconnection Features and Related Parameters, Proceedings of the 2001 IEEE International Conference on Microelectronic Test Structures, Kobe, Japan, Mar. 19-22, 2001, vol. 14, pp. 183-188.

3. Cresswell, M. W., Bonevich, J. E., Allen, R. A., Guillaume, N. M. P., Giannuzzi, L. A., Everist, S. C., Murabito, C. E., Shea, P. J., and Linholm, L. W., Electrical Linewidth Test Structures Patterned in (100) Silicon-on-Insulator for Use as CD Standards, IEEE Transactions on Semiconductor Manufacturing, vol. 14, no. 4, 2001, p9.

Alignment with NSET Grand Challenges

\begin{tabular}{|c|c|c|c|c|c|c|c|c|c|c|}
\hline MAT & ELEC & HEALTH & ENV & ENER & SPACE & BIOSEN & TRANS & SECUR & MET & MAN \\
\hline & $\mathrm{X}$ & & & & & & & & $\mathrm{X}$ & \\
\hline
\end{tabular}




\section{Electronics and Electrical Engineering Laboratory}

\section{Nanostructure Fabrication and Metrology}

\section{Project Leader/contact:}

Rich Mirin, 303-497-7955, mirin@boulder.nist.gov

Optoelectronics Division, EEEL, Boulder

Approximate staffing:

6.2 FTE

Funding sources:

NIST $(100 \%)$

Objective:

Develop measurement technology and methods based on epitaxial semiconductor nanostructures. Develop new techniques to fabricate, characterize and model nanostructures.

Constituency:

Optoelectronics industry, nanoscale metrology equipment developers, and measurement instrumentation manufacturers

Principal current tasks:

- Generate single photons on demand (single photon turnstile)

- Capacitance spectroscopy of ensembles of quantum dots and individual quantum dots

- Single photon detectors based on quantum dots

- Single quantum dot absorption measurements

- Etch 2D and 3D photonic crystals using chemically-assisted ion beam etching

- Model photonic crystals

- Measure and model quantum dot, semiconductor optical amplifiers

- Molecular beam epitaxy of self-assembled quantum dots

Additional information:

NIST is developing the fabrication and metrology of compound semiconductor nanostructures to support the optoelectronics industry in this area. Control and measurement of the size, location, and optical properties of nanostructures are fundamental to their applications. For example, the resonant absorption cross-sections and the homogeneous linewidths of quantum dots have not yet been measured. Single photon generation and detection based on single quantum dots is being pursued. The ultrafast properties of quantum dots in a waveguide are being studied for the realization of the next-generation of optical devices such as laser diodes, photodetectors, and optical amplifiers. Photonic crystals for control of the single photons are being investigated.

Selected publications:

1. R.P. Mirin, K.L. Silverman, D.H. Christensen, and A. Roshko, "Narrow Photoluminescence Linewidths From Ensembles of Self-Assembled InGaAs Quantum Dots", Journal of Vacuum Science and Technology. B 18 (3), 1510-1513 (2000).

2. R.P. Mirin, A.C. Gossard, and J.E. Bowers, "Characterization of InGaAs quantum dot lasers with a single quantum dot layer as an active region", Physica E 2 (1-4), 738-742 (1998).

Alignment with NSET Grand Challenges:

\begin{tabular}{|c|c|c|c|c|c|c|c|c|c|c|}
\hline MAT & ELEC & HEALTH & ENV & ENER & SPACE & BIOSEN & TRANS & SECUR & MET & MAN \\
\hline $\mathrm{X}$ & $\mathrm{X}$ & & & & & & & & $\mathrm{X}$ & \\
\hline
\end{tabular}




\section{Electronics and Electrical Engineering Laboratory}

\section{Semiconductor Nanostructure Arrays}

Project Leader/contact:

Kris Bertness, 303-497-5069, bertness@boulder.nist.gov

Optoelectronics Division, EEEL, Boulder

Approximate staffing:

0.7 FTE

Funding sources:

NIST (20\%), Other Agency (80\%)

Objective:

Develop methods to grow and characterize quantum dots on nanopatterned substrates.

Constituency:

Optoelectronics industry, epitaxial crystal growth industry

Principal current tasks:

- Optimize surface preparation and cleaning for silicon and GaP surfaces that preserve substrate nanopatterns.

- Develop RHEED assessment of surface preparation and quantum dot formation.

- Develop growth procedures for regular array formation on nanopatterned substrates.

Additional information:

NIST is collaborating through a cooperative research and development agreement (CRADA) with Systine, Inc., Pasadena, CA, to develop methods for the fabrication of high-quality, high-density arrays of semiconductor quantum dots. This research, if successful, will be applicable to a myriad of optoelectronic applications, including infrared detection and visible light displays. The technology makes use of biologically generated patterns and a patented low-energy electron-enhanced etching technique.

Alignment with NSET Grand Challenges:

\begin{tabular}{|c|c|c|c|c|c|c|c|c|c|c|}
\hline MAT & ELEC & HEALTH & ENV & ENER & SPACE & BIOSEN & TRANS & SECUR & MET & MAN \\
\hline $\mathrm{X}$ & $\mathrm{X}$ & & & & & & & & $\mathrm{X}$ & \\
\hline
\end{tabular}




\section{Electronics and Electrical Engineering Laboratory}

\section{Semiconductor Nanoscale Imaging and Analysis}

\section{Project Leader/contact:}

- Alexana Roshko, 303-497-5420, roshko@boulder.nist.gov

Optoelectronics Division, EEEL, Boulder

- Robert Keller, 303-497-7651, keller@boulder.nist.gov

Materials Reliability Division, MSEL, Boulder

- John E. Bonevich, 301-975-5428, john.bonevich@ nist.gov

Metallurgy Division, MSEL, Gaithersburg

Approximate staffing:

2.9 FTE

Funding sources:

NIST $(100 \%)$

Objective:

Develop methods to image and characterize compound semiconductors on a nanometer length scale.

Constituency:

Optoelectronics industry.

Principal current tasks:

- $\quad$ Atomic force microscopy (AFM) and transmission electron microscopy (TEM) imaging of nanoscale structures, such as quantum dot dimensions and distributions.

- Develop convergent beam electron diffraction (CBED) imaging and analysis for measurement of strain in compound semiconductors.

- Analyze strain adjacent thin buried native oxide layers; evaluate methods of strain minimization.

- Determine relationships between strain and quantum dot morphology.

Additional information:

The abilities to image nanoscale features and to map strain in nanoscale structures are crucial to optoelectronic device development where structures on the order of $2 \mathrm{~nm}$ to $100 \mathrm{~nm}$ in dimension are commonplace. AFM and TEM imaging are being used in tandem to measure the size, shape and distribution of quantum-scale structures. This information is then correlated with CBED measurements of strain in these materials to predict optimal growth conditions for obtaining desired morphologies. Currently under investigation are self-assembled quantum dots and native oxide layers formed by wet-thermal oxidation of AlGaAs. The native oxide work for VCSEL applications is part of a collaboration with a major optoelectronics manufacturer and a national laboratory.

Alignment with NSET Grand Challenges:

\begin{tabular}{|l|l|l|l|l|l|l|l|l|l|l|}
\hline MAT & ELEC & HEALTH & ENV & ENER & SPACE & BIOSEN & TRANS & SECUR & MET & MAN \\
\hline $\mathrm{X}$ & $\mathrm{X}$ & & & & & & & & $\mathrm{X}$ & $\mathrm{X}$ \\
\hline
\end{tabular}




\section{Electronics and Electrical Engineering Laboratory}

\section{Nanoscopic Nonlinear Optical and Spectroscopic Metrologies in Wide-Bandgap Semiconductors}

\section{Project Leader/contact:}

Norman A. Sanford, 303-497-5239, sanford@ boulder.nist.gov

Optoelectronics Division, EEEL, Boulder

Approximate staffing:

1.8 FTE (plus coordinated efforts with MSEL and CSTL)

Funding sources:

NIST $(100 \%)$

Objective:

Develop correlated nanoscopic nonlinear optical and spectroscopic methods to examine the optical, structural, and electronic properties of defects in wide-bandgap III-nitride semiconductors.

Constituency:

Optoelectronics industry, high-power electronics industry

Principal current tasks:

- Develop blue/uv, collection-mode, near-field spectroscopy with $50 \mathrm{~nm}$ spatial resolution

- Develop collection-mode, near-field, nonlinear optics in the blue/uv spectral range

- Investigate the structural and electronic properties of defects in III-nitride semiconductors

- Examine the electronic properties of defects in the vicinity of ohmic contacts

\section{Additional information:}

The wide-bandgap III-nitride semiconductors are important emerging materials for the development of solid-state lighting, blue/green lasers for optical data storage, and high-power, high-temperature electronic devices. The potential market represented by worldwide adoption of LED-based solid-state lighting is projected to be $\$ 50$ billion dollars per year over the next few decades. Nevertheless, all of the technologies enabled by the wide-bandgap IIInitrides are impeded in their development due to poor fundamental understanding of the role of defects in the optical, structural, and electronic properties of these materials. The development of new, correlated, nanoscale metrologies that address these issues is critical for progress in the material system. This effort is highly collaborative with NIST staff in MSEL and CSTL who bring expertise in x-ray, TEM, SEM, cathodoluminescence, metallurgy, and various analytical methods.

Alignment with NSET Grand Challenges:

\begin{tabular}{|c|c|c|c|c|c|c|c|c|c|c|}
\hline MAT & ELEC & HEALTH & ENV & ENER & SPACE & BIOSEN & TRANS & SECUR & MET & MAN \\
\hline $\mathrm{X}$ & $\mathrm{X}$ & & & & & & & & $\mathrm{X}$ & \\
\hline
\end{tabular}




\section{Electronics and Electrical Engineering Laboratory}

\section{Nanoscale Quantum Standards: Capacitance and Current Standards Based On Counting Electrons}

Project Leader/contact:

- Mark Keller, 303-497-430, mark.keller@boulder.nist.gov

Electromagnetic Technology Division, EEEL, Boulder

- Neil Zimmerman, 301-975-5887, neil.zimmerman@ nist.gov

Electricity Division, EEEL, Gaithersburg

Approximate staffing:

3.5 FTE

Funding sources

NIST (80\%), Other Agencies (20\%)

Objective:

To develop new fundamental standards of capacitance and current based on a fundamental property of nature, the electron charge, by exploiting the ability of single-electron tunneling (SET) devices to manipulate

individual electrons at $\mathrm{MHz}$ frequencies through nanoscale tunnel junctions.

Constituency:

This project is aimed at providing improved capacitance calibration services to NIST customers in industry and at offering a new basis for standards of capacitance and current to the community of metrologists interested in electrical metrology and fundamental constants experiments.

Principal current tasks:

- Compare the existing prototype electron counting capacitance standard with NIST's established primary standard to determine the level of agreement between the two.

- Develop a current standard that delivers of order $100 \mathrm{pA}$ to enable an experiment known as the quantum metrology triangle that will contribute to our understanding of the three existing quantum electrical standards for current, voltage, and resistance.

Additional information:

Single-electron tunneling devices containing nanoscale tunnel junctions allow electrons to be moved one at a time in response to applied gate voltages. The capacitance and current standards use a device known as an electron pump that can pass large numbers of electrons with an uncertainty approaching 1 part per billion. The current standard is created by simply driving the gate voltages at a frequency locked to an atomic clock or other accurate frequency standard. The current produced by the present electron pump is limited to about $1 \mathrm{pA}$, but we are exploring other SET technologies to push toward much larger currents. The capacitance standard operates by placing a known number of electrons onto a capacitor and measuring the resulting voltage, and the capacitance is simply the ratio of charge to voltage. A prototype capacitance standard based on counting electrons has been demonstrated to have an uncertainty of less than $1 \mathrm{ppm}$.

\section{Selected publications:}

1. M. W. Keller, A. L. Eichenberger, J. M. Martinis, and N. M. Zimmerman, "A Capacitance Standard Based on Counting Electrons," Science 285, 1706 (1999).

2. M. W. Keller, "Standards of Current and Capacitance Based on Single-Electron Tunneling Devices," to appear in proceedings of Fermi School CXLVI: "Recent Advances in Metrology and Fundamental Constants," 2001.

Alignment with NSET Grand Challenges:

\begin{tabular}{|c|c|c|l|l|l|l|l|c|c|c|}
\hline MAT & ELEC & HEALTH & ENV & ENER & SPACE & BIOSEN & TRANS & SECUR & MET & MAN \\
\hline & $\mathrm{X}$ & & & & & & & $X$ & $X$ & $X$ \\
\hline
\end{tabular}




\section{Electronics and Electrical Engineering Laboratory}

\section{Nanoscale Quantum Standards: \\ Arbitrary Waveform Generators for Standards, Communications, and Radar}

\section{Project Leader/contact:}

Sam Benz, 301-497-5258, benz@boulder.nist.gov

Electromagnetic Technology Division, EEEL, Boulder

Approximate staffing:

2.0 FTE

Funding sources

NIST (40\%), Other Agencies (60\%)

Objective:

To develop high performance superconducting integrated circuits and systems for high precision voltage metrology at NIST and the electronics and communications industries and for low-phase-noise radar.

Constituency:

This project addresses voltage metrology needs for NIST and the electronic instrumentation and communications industries as well as defense department interests in developing the next generation of advanced digital radar and communication systems.

Principal current tasks:

- Develop nanoscale junction fabrication techniques for lumped arrays of Josephson junctions with spacing between junctions of $10 \mathrm{~nm}$ to $100 \mathrm{~nm}$.

\section{Additional information:}

Our joint invention with Northrop Grumman for a Josephson arbitrary waveform synthesizer is applicable to radar, communications and metrology applications. Only superconducting Josephson junctions can provide the perfect quantization necessary for producing the precise voltage amplitudes, low phase noise, and high spur-free dynamic range of an ideal digitally synthesized waveform. Because of the small voltages produced by single junctions, full realization of these features for practical systems hinges on the development of lumped arrays. An array is considered lumped when all of the junctions act in unison as a single element of a microwave circuit. This is accomplished only when the total array length with approximately 15,000 junctions is a small fraction (typically 1 $\mathrm{mm}$ ) of the wavelength of the highest microwave drive frequency. Present Josephson junction fabrication techniques are capable of junction spacing of $5 \mu \mathrm{m}$ to $10 \mu \mathrm{m}$. In order to achieve the 100- to 1000 -fold decrease in junction spacing, novel fabrication methods are necessary. We are presently investigating a number of techniques for fabricating nanoscale junctions that require three-dimensional nanoscale control of the junction and array dimensions.

\section{Selected publications:}

1. S.P. Benz, P.D. Dresselhaus, and C.J. Burroughs, Nanotechnology for next generation Josephson voltage standards, Proceedings of the Symposium on Microtechnology in Metrology and Metrology in Microsystems, 31 August-1 September 2000, Delft University of Technology, Delft, The Netherlands, pp. 127-132.

2. S.P. Benz, C.J. Burroughs, and P.D. Dresselhaus, Low harmonic distortion in a Josephson arbitrary waveform synthesizer, Applied Physics Letters Vol. 77, No. 7, pp. 1014-1016 (14 August 2000).

Alignment with NSET Grand Challenges:

\begin{tabular}{|c|c|c|l|l|l|l|l|c|c|c|}
\hline MAT & ELEC & HEALTH & ENV & ENER & SPACE & BIOSEN & TRANS & SECUR & MET & MAN \\
\hline & $\mathrm{X}$ & & & & & & & $\mathrm{X}$ & $\mathrm{X}$ & $\mathrm{X}$ \\
\hline
\end{tabular}




\section{Electronics and Electrical Engineering Laboratory}

\section{Nanoscale Quantum Standards: Radiometry Standard Based on Counting Photons}

\section{Project Leader/contact:}

- Richard Mirin, 303-497-7955, mirin@boulder.nist.gov

Optoelectronics Division, EEEL, Boulder

- Mark Keller, 303-497-5430, mark.keller@boulder.nist.gov

Electromagnetic Technology Division, EEEL, Boulder

Approximate staffing:

$2.5 \mathrm{FTE}$

Funding sources

NIST (100\%),

Objective:

To develop a source of individual photons controlled with an applied voltage by exploiting the unique optical and electrical properties of nanoscale semiconductor quantum dots.

Constituency:

This project is aimed at providing a new quantum standard of optical intensity near the commercially important wavelength of $1.5 \mu \mathrm{m}$, so it is expected to benefit fundamental optical metrology as well as users of NIST's optical calibration services in industry.

Principal current tasks:

- Demonstrate a single-photon turnstile based on a single quantum dot embedded in an appropriate semiconductor structure.

Additional information:

A fundamental property of light is that it is quantized in photons, and a conceptually simple standard for optical intensity is a device that generates individual photons at a known rate. The first realization of this simple scheme with metrological accuracy is the object of this project. A semiconductor quantum dot can be made so small (on the scale of $20 \mathrm{~nm}$ ) that individual electron and hole states in the dot can be addressed separately. When a quantum dot is loaded with one electron and one hole, the two recombine and produce a photon. In a single-photon turnstile, the injection of electrons and holes into a quantum dot, and thus the production of individual photons, is controlled by an applied voltage. Combining expertise in nanoscale quantum dot growth with expertise in manipulating individual electrons, this project is working toward a goal of making a single-photon turnstile that has a repetition rate of order $1 \mathrm{GHz}$ and an error rate of less than 1 per million cycles.

Alignment with NSET Grand Challenges:

\begin{tabular}{|c|c|c|l|l|l|l|l|c|c|c|}
\hline MAT & ELEC & HEALTH & ENV & ENER & SPACE & BIOSEN & TRANS & SECUR & MET & MAN \\
\hline $\mathrm{X}$ & $\mathrm{X}$ & & & & & & & $\mathrm{X}$ & $\mathrm{X}$ & \\
\hline
\end{tabular}




\section{Electronics and Electrical Engineering Laboratory}

\section{Nanoscale Quantum Standards: Quantum Time Standards and Computing (Fabrication)}

Project Leader/contact:

- Jim Beall, 303-497-5989, james.beall@boulder.nist.gov

Electromagnetic Technology Division, EEEL, Boulder

- David Wineland, 303-497-5286,david.wineland@boulder.nist.gov

Time and Frequency Division, Physics Laboratory, Boulder

Approximate staffing:

1.0 FTE

Funding sources

NIST $(100 \%)$

Objective:

Develop microfabricated ion trap structures for use in experiments in timekeeping, quantum logic, quantum decoherence, and quantum measurement theory.

Constituency:

This work promises to improve timekeeping and its myriad important applications, such as the global positioning system, and to determine the feasibility of quantum computing.

Principal current tasks:

- Fabrication of micromachined ion traps, and arrays of them, in collaboration with the Time and Frequency Division.

\section{Additional information:}

A collaborative program between EEEL and PL to develop microfabrication techniques for ion confinement structures that allow tight confinement, optical access for laser cooling and probing, and advanced electrode configurations for multiple ion manipulation.

Selected publications:

C. A. Sackett, D. Kielpinski, B. E. King, C. Langer, V. Meyer, C. J. Myatt, M. Rowe, Q. A. Turchette, W. M.

Itano, D. J. Wineland, and C. Monroe, "Experimental entanglement of four particles," Nature 404, 256 (2000).

Alignment with NSET Grand Challenges:

\begin{tabular}{|c|c|l|l|l|l|l|l|c|c|c|}
\hline MAT & ELEC & HEALTH & ENV & ENER & SPACE & BIOSEN & TRANS & SECUR & MET & MAN \\
\hline & $\mathrm{X}$ & & & & & & & $X$ & $X$ & \\
\hline
\end{tabular}




\section{Electronics and Electrical Engineering Laboratory}

\section{X-ray Microanalysis of Nanometer Scale Particles and Films}

\section{Project Leader/contact:}

Kent Irwin, 303-497-5911, kent.irwin@boulder.nist.gov

Electromagnetic Technology Division, EEEL, Boulder

Approximate staffing:

6.0 FTE

Funding sources

NIST (40\%), Other Agencies (60\%)

Objective:

To develop a new class of $\mathrm{x}$-ray detectors capable of detecting photons with high-energy resolution and nearunity quantum efficiency that will enable new generations of spectroscopic tools to be created.

Constituency:

This project is aimed at providing x-ray microanalysis tools needed to evaluate nanometer-scale structures in any materials intensive industry. A key initial adopter will be the semiconductor manufacturing industry, where identification of nanometer-sized particles and thin film structures is critical.

Principal current tasks:

- Continue the development of the single pixel x-ray detector systems for transfer to CSTL in NIST and to the U.S. industry licensees of the technology.

- Develop arrays of detectors and associated readout electronics to dramatically increase the collection area and count rate of the spectrometer.

Additional information:

The ability to analyze the chemical composition of nanometer size particles and nanometer thick thin films is critical to a variety of materials intensive industries, including the semiconductor manufacturing industry. The development of cryogenic microcalorimeter detectors has produced a new class of energy dispersive x-ray spectrometers that allow this type of analysis to be performed quickly and reliably for the first time. Introducing a radically new technology such as cryogenic microcalorimeters to a large community requires creating and demonstrating an entire measurement instrument, not just the detector. In addition to developing revolutionary $\mathrm{x}$-ray detectors, we have developed superconducting electronics to read the detectors, compact adiabatic demagnetization refrigerators to simplify cooling the detectors to millikelvin operating temperatures, and room-temperature electronics to process the output signals.

\section{Selected publications:}

1. Geer, R.E.; Wu, D.; Wollman, D.A., "High-resolution EDS Analysis of Ultra-Thin TaSiN Diffusion Barriers for Cu Metallization Using Microcalorimetry", Proc. IEEE 2001 Int'l Interconnect Tech. Conf., 192-194 (2001).

2. Wollman, D.A.; Nam, S.W.; Hilton, G.C.; Irwin, K.D.; Bergren, N.F.; Rudman, D.A.; Martinis, J.M.; Newbury,D.E., "Microcalorimeter Energy-Dispersive Spectrometry Using a Low Voltage Scanning Electron Microscope," J. Microscopy 199, 37-44 (2000).

3. C. K. Stahle, D. McCammon, K. D. Irwin, "Quantum Calorimetry," Physics Today 52, 32 (1999).

Alignment with NSET Grand Challenges:

\begin{tabular}{|c|c|c|c|c|c|c|c|c|c|c|}
\hline MAT & ELEC & HEALTH & ENV & ENER & SPACE & BIOSEN & TRANS & SECUR & MET & MAN \\
\hline & $\mathrm{X}$ & & & & & & & & $\mathrm{X}$ & $\mathrm{X}$ \\
\hline
\end{tabular}




\section{Electronics and Electrical Engineering Laboratory}

\section{Electrical Measurements of Single Molecular Rotors}

Project Leader/contact:

Mark Keller, 303-497-5430, mark.keller@boulder.nist.gov

Electromagnetic Technology Division, EEEL, Boulder

Approximate staffing:

$0.5 \mathrm{FTE}$

Funding sources

NIST $(100 \%)$

Objective:

To develop a new probe of single molecules by exploiting the sensitivity of single-electron tunneling transistors to electrical charge.

Constituency:

This project is aimed at providing a new tool for characterizing charge motion in single molecules and is expected to have impact across much of the field of molecular (or other nanoscale) electronics.

Principal current tasks:

- Use a single-electron tunneling transistor to detect changes in polarization caused by the rotation of molecules having a permanent electric dipole.

Additional information:

There is a class of molecules known as rotors that consist of a shaft attached to a substrate and a rotating part that is permanently charged with opposite polarity on its two ends. The details of how these molecules rotate and how ensembles of molecules interact are currently not well understood. A single-electron tunneling transistor containing nanoscale tunnel junctions can detect a charge as small as one thousandth of an electron charge, making it suitable for studying the motion of these rotor molecules either singly or in ensembles. This new tool will find uses in a variety of ways that will benefit the overall development of electronics based on molecules or other nanoscale objects.

Alignment with NSET Grand Challenges:

\begin{tabular}{|c|c|c|l|l|l|c|c|c|c|c|}
\hline MAT & ELEC & HEALTH & ENV & ENER & SPACE & BIOSEN & TRANS & SECUR & MET & MAN \\
\hline $\mathrm{X}$ & $\mathrm{X}$ & $\mathrm{X}$ & & & & $\mathrm{X}$ & & & $\mathrm{X}$ & $\mathrm{X}$ \\
\hline
\end{tabular}




\section{Electronics and Electrical Engineering Laboratory}

\section{Rectification of Light as an Electrical Power Source}

\section{Project Leader/contact:}

Erich Grossman, 303-497-5102, erich.grossman@boulder.nist.gov

Electromagnetic Technology Division, EEEL, Boulder

Approximate staffing:

1.0 FTE

Funding sources

NIST $(100 \%)$

Objective:

To demonstrate direct rectification of near-IR radiation to DC in an antenna-coupled nanodiode, and to analyze the performance of nanodiodes for electrical power generation from solar radiation or "waste" thermal radiation.

Constituency:

This project addresses the long-term national need for alternative electrical power sources.

Principal current tasks:

- Demonstrate photon-assisted tunneling (at room temperature) in a nanodiode illuminated by near-IR radiation.

\section{Additional information:}

The ability to analyze the chemical composition of nanometer size particles and nanometer thick thin films is critical to a variety of materials intensive industries, including the semiconductor manufacturing industry. The development of cryogenic microcalorimeter detectors has produced a new class of energy dispersive x-ray spectrometers that allow this type of analysis to be performed quickly and reliably for the first time. Introducing a radically new technology such as cryogenic microcalorimeters to a large community requires creating and demonstrating an entire measurement instrument, not just the detector. In addition to developing revolutionary x-ray detectors, we have developed superconducting electronics to read the detectors, compact adiabatic demagnetization refrigerators to simplify cooling the detectors to millikelvin operating temperatures, and room-temperature electronics to process the output signals.

Alignment with NSET Grand Challenges:

\begin{tabular}{|c|c|c|c|c|l|l|l|l|l|l|}
\hline MAT & ELEC & HEALTH & ENV & ENER & SPACE & BIOSEN & TRANS & SECUR & MET & MAN \\
\hline & $\mathrm{X}$ & & & $\mathrm{X}$ & & & & & & \\
\hline
\end{tabular}




\section{Electronics and Electrical Engineering Laboratory}

\section{Nanomagnetodynamics: Materials Metrology for Data Storage}

\section{Project Leader/contact:}

- Tom Silva, 303-497-7826, silva@ boulder.nist.gov

Magnetic Technology Division, EEEL, Boulder

- Bob McMichael, 301-975-5121, robert.mcmichael@nist.gov

Metallurgy Division, MSEL, Gaithersburg

- Donna Hurley, 303-497-3081, hurley@boulder.nist.gov

Materials Reliability Division, MSEL, Boulder

Approximate staffing:

2.5 FTE (Div 816)

Funding sources:

NIST (77 \%), Other Agencies (23\%)

Objective:

Develop tools to permit data to be stored at speeds approaching the fundamental gyromagnetic quantum limit and at densities of almost one terabit per square inch ( 1 bit at $25 \mathrm{~nm}$ by $25 \mathrm{~nm}$ ).

Constituency:

Magnetic data storage industry, university research

Principal current tasks

- Develop a ferromagnetic resonance (FMR) spectrometer with sensitivity at the level of 10,000 Bohr magnetons based on micro-electromechanical systems (MEMS)

- Develop a frequency-swept $(4 \mathrm{GHz}-10 \mathrm{GHz})$ ferromagnetic resonance spectrometer

- Develop and apply a broadband $(2 \mathrm{GHz}-4 \mathrm{GHz})$ Michelson interferometer for the detection of magnon-phonon scattering processes

- Develop a scanned giant-magnetoresistance (GMR) probe microscope with $10 \mathrm{~nm}$ spatial resolution and $50 \mathrm{ps}$ time resolution.

\section{Additional information:}

There is a need to understand and control the atomic-scale processes that dissipate spin excitations in ferromagnetic systems. This dissipation process, or damping, governs the switching speed of magnetic memory element by coupling the intrinsic angular momentum of the magnetic spins to the crystal lattice. Data rates for commercial disk drives must increase to account for the ever-increasing linear bit densities. Linear densities are currently at $100 \mathrm{~nm}$ per bit. In two years, this will shrink to $50 \mathrm{~nm}$. Such densities will require operational bandwidths in excess of 1.4 $\mathrm{GHz}$ in the near future. Researchers in the magnetic data storage industry urgently need pre-competitive assistance to comprehend damping as bandwidths approach $1 \mathrm{GHz}$. The emergent magnetic random access memory (MRAM) industry is also in need of tools for the control of magnetic damping. To be competitive with semiconductor static random access memory (SRAM), MRAM data rates must be in excess of 1 gigabit per second.

Alignment with NSET Grand Challenges

\begin{tabular}{|c|c|c|c|c|c|c|c|c|c|c|}
\hline MAT & ELEC & HEALTH & ENV & ENER & SPACE & BIOSEN & TRANS & SECUR & MET & MAN \\
\hline $\mathrm{X}$ & $\mathrm{X}$ & & & & & & & & $\mathrm{X}$ & \\
\hline
\end{tabular}




\section{Electronics and Electrical Engineering Laboratory}

\section{Single Molecule Manipulation and Measurement $\left(\mathrm{SM}^{3}\right)$}

\section{Project Leader/contact:}

- John Moreland, 303-497-3641, moreland@boulder.nist.gov

Magnetic Technology Division, EEEL, Boulder

- John Kasianowicz, 301-975-5853, john.kasianowicz@ nist.gov

Biotechnology Division, CSTL, Gaithersburg

- Angela Hight Walker, 301-975-2155, angela.hightwalker@nist.gov

Optical Technology Division, PL, Gaithersburg

Approximate staffing:

0.33 FTE (Div 816 only)

\section{Funding sources:}

NIST (100\%)

Objective:

Develop single-molecule technologies to address significant inaccuracies in the base-pair ordering inferred by present gene-sequencing tools, affecting such well-publicized efforts as the Human Genome Project.

\section{Constituency:}

Health care industries, advanced materials industries, university research

Principal current tasks:

- Develop fabrication methods for nanometer-scale elements and fluidic systems that will transport single molecules

- Develop measurement methods for structural properties of deoxyribonucleic acid (DNA) using pores

- Develop the ability to interpret fluorescence resonance energy transfer (FRET) data unambiguously on single molecules and methods to measure the structure and dynamics of ribonucleic (RNA) standards and hairpin systems

- Develop the ability to measure single molecules spectroscopically, using surface enhanced Raman spectroscopy (SERS) and optically, using two-photon and UV excitation with simultaneous force measurements

\section{Additional information:}

The program will ensure the integrity of such bio-informatic databases through the development and utilization of a high-throughput, single molecule manipulation and measurement $\left(\mathrm{SM}^{3}\right)$ platform to directly manipulate and measure the structure and dynamics of single RNA or DNA molecules. Within the five-year time span of this effort, the following will be developed: A platform will be produced that will combine these capabilities to make a single biomolecule measurement workbench.

Alignment with NSET Grand Challenges:

\begin{tabular}{|c|c|c|c|c|c|c|c|c|c|c|}
\hline MAT & ELEC & HEALTH & ENV & ENER & SPACE & BIOSEN & TRANS & SECUR & MET & MAN \\
\hline $\mathrm{X}$ & & $\mathrm{X}$ & & & & & & & $\mathrm{X}$ & \\
\hline
\end{tabular}




\section{Electronics and Electrical Engineering Laboratory}

\section{Coherent Spin Electronics for Microwave and Terahertz Communication and Signal Processing}

\section{Project Leader/contact:}

Stephen Russek, 303-497-5097, russek@boulder.nist.gov

Magnetic Technology Division, EEEL, Boulder

Approximate staffing:

3 FTE

Funding sources:

NIST ( $85 \%)$, Other Agencies (15\%)

Objective:

Metrology and understanding of high frequency dynamics in magnetic materials.

Constituency:

Telecommunication industries, magnetic storage industries, university research

Principal current tasks:

- Measure high-frequency spin precession and resonance in a two-dimensional degenerate electron gas (2DEG)

- Examine the interaction of the electron spins in a ferromagnetic layer with spins in a semiconductor substrate under different electrical excitations and transport conditions in order to understand the depolarization process

\section{Additional information:}

Devices employing electrically excited coherent spin precession ( $1 \mathrm{GHz}$ to $1 \mathrm{THz}$ ) will be developed. The key innovation will be the use of the recently discovered spin-momentum-transfer (SMT) effect to enable electrical manipulation of spins in nanoscale devices, rather than the use of applied magnetic fields or polarized light pulses. The work is in collaboration with Cornell University and Motorola Research Labs. NIST will concentrate on the metrology and understanding of high frequency dynamics in magnetic materials. In the SMT phenomenon, a dc spinpolarized electron current incident on a magnetic element provides a direct transfer of spin angular momentum in the process of scattering, thereby imparting a torque on the magnetization of the active element. At sufficiently large currents, stimulated emission ensues, resulting in uniform spin precession within the ferromagnet and the potential for diffusion of coherent spins into adjacent contacts. This effect will be applied to develop spin-coherent devices using hybrid ferromagnet/semiconductor heterostructures in which the precessional dynamics of excited spins are exploited for applications in high-speed communications and signal processing.

Alignment with NSET Grand Challenges

\begin{tabular}{|c|c|c|c|c|c|c|c|c|c|c|}
\hline MAT & ELEC & HEALTH & ENV & ENER & SPACE & BIOSEN & TRANS & SECUR & MET & MAN \\
\hline & $\mathrm{X}$ & & & & & & & & $\mathrm{X}$ & \\
\hline
\end{tabular}




\section{Electronics and Electrical Engineering Laboratory}

\section{Nanoprobe Imaging}

\section{Project Leader/contact:}

John Moreland, 303-497-3641, moreland@boulder.nist.gov

Magnetic Technology Division, EEEL, Boulder

Approximate staffing:

$0.5 \mathrm{FTE}$

Funding sources:

NIST $(100 \%)$

Objective:

Development of scanned-probe microscopy (SPM) and micro-electromechanical systems (MEMS) for nanometer-scale magnetic measurements in support of the magnetic data storage industry.

Constituency:

Magnetic Storage Industry

Principal current tasks:

- Development of a "magnetic resonance spectrometer on a chip" to achieve magnetic-resonance imaging resolution of $1 \mathrm{~nm}$ on ferromagnetic thin films.

\section{Additional information:}

Project members perform research to understand and relate SPM images and MEMS magnetometer measurements to the performance of magnetic materials and devices for future recording technologies. The project is currently focusing on ultra-small magnetic-force microscopy tips for imaging recording heads and media at a resolution of $20 \mathrm{~nm}$. Quantitative field mapping of heads and media is based on electromechanical detection of magnetic resonance. MEMS magnetometers with integrated specimens and high sensitivity are being developed. Over the next few years, the project will work on a "magnetic resonance spectrometer on a chip" to achieve magnetic-resonance imaging resolution of $1 \mathrm{~nm}$ on ferromagnetic thin films. Recent research includes the development of new ferromagnetic resonance (FMR) spectrometers based on calorimetry, torque, and transfer of spin angular momentum. Such sensors can be integrated with atomic-force microscopes for imaging of local dc and rf magnetic fields.

\section{Selected publications:}

1. Markus Lohndorf, John Moreland, Pavel Kabos, and Nick Rizzo, "Microcantilever torque magnetometry of thin magnetic films," J. Appl. Phys. 87, pp. 5995-5997 (2000).

2. John Moreland, Markus Lohndorf, Pavel Kabos, and R. D. McMichael, "Ferromagnetic resonance spectroscopy with a micromechanical calorimeter sensor," Rev. Sci. Instrum. 8, 3099 (2000).

3. Albrecht Jander, John Moreland, and Pavel Kabos, "Micromechanical Detectors for Local Field Measurements Based on Ferromagnetic Resonance," J. Appl. Phys. 89, 7086 (2001)

Alignment with NSET Grand Challenges:

\begin{tabular}{|c|c|c|c|c|c|c|c|c|c|c|}
\hline MAT & ELEC & HEALTH & ENV & ENER & SPACE & BIOSEN & TRANS & SECUR & MET & MAN \\
\hline & $\mathrm{X}$ & & & & & & & & $\mathrm{X}$ & \\
\hline
\end{tabular}




\section{Electronics and Electrical Engineering Laboratory}

\section{Emerging Applications of Nanoscale Magnetoresistive Sensors: Forensics, Non-destructive Failure Analysis, Surveillance, Biological Monitoring}

\section{Project Leader/contact:}

- David P. Pappas, 303-497-3374, david.pappas@ nist.gov

Magnetic Technology Division, EEEL, Boulder

Approximate staffing:

$1.5 \mathrm{FTE}$

Funding sources:

NIST (83\%), Other Agencies (17\%)

\section{Objective:}

Map out magnetic fields with high sensitivity using room temperature magnetoresistive arrays. The target sensitivity is different for the various applications such as forensics analysis and bio-magnetic field sensors. In general, we will be advancing the area of magnetic field metrology by leveraging off of the rapid development of magnetic sensors seen in the data storage industry.

\section{Constituency:}

Forensics analysts, security (detection of low levels of ferrous materials), bio-magnetism (electrocardiograph, ECG, and possibly electroencephalograph, EEG, monitoring), sensors for monitoring personnel and equipment movement in military settings, sensors for satellite magnetic field measurements, VLSI (very large scale integration) device and packaging failure analysis.

\section{Principal current tasks:}

- Develop a real-time imaging system for analog storage media analysis.

- Extract valuable information from damaged/modified digital magnetic data storage media.

- Map out fields and invert Maxwell's equations to identify sources of leakage currents in VLSI chips for failure analysis.

\section{Additional information:}

High sensitivity magnetic field measurement is important in a wide range of activities. We have been developing scanning elements and arrays of elements to map out the spatial variations of magnetic fields with high resolution. As we develop higher sensitivities and spatial resolution, we find more and more applications of these devices. For low field measurements, the present technology uses super-conducting devices that must be held at low temperature. We are finding that as the understanding of magnetoresistive materials evolves and the development of magnetic flux concentrators is incorporated, devices that bring these technologies together can compete with the low temperature sensors.

\section{Selected publications:}

D. P. Pappas, et al. "Second Harmonic Magneto-Resistive Imaging to Authenticate and Recover Data from Magnetic Storage Media" Proceedings of SPIE Conference on Technologies for Law Enforcement, Boston, MA, in press, Nov. 2000.

Alignment with NSET Grand Challenges

\begin{tabular}{|c|c|c|c|c|c|c|c|c|c|c|}
\hline MAT & ELEC & HEALTH & ENV & ENER & SPACE & BIOSEN & TRANS & SECUR & MET & MAN \\
\hline $\mathrm{X}$ & $\mathrm{X}$ & $\mathrm{X}$ & & & $\mathrm{X}$ & $\mathrm{X}$ & & $\mathrm{X}$ & $\mathrm{X}$ & \\
\hline
\end{tabular}




\section{Manufacturing Engineering Laboratory}

\section{Atom-scale Length Measurement, Standards Fabrication, and Nanomanufacturing}

\section{Project Leader/contact:}

Rick Silver, 301-975-5609, richard.silver@ nist.gov

Precision Engineering Division, MEL, Gaithersburg

Approximate staffing:

4.0 FTE

Funding sources

NIST (95\%), International SEMATECH (5\%)

Objective:

To calibrate atomically-defined, three-dimensional length standards for nanomanufacturing and related industries.

Constituency:

Semiconductor electronics, magnetic storage, micro electromechanical systems (MEMS), and emerging nanotechnology industries

Principal current tasks:

- Currently writing features in silicon with critical dimensions as small as $10 \mathrm{~nm}$. Improve the fabrication robustness to enable the regular processing of features this size and smaller.

- Use the field ion microscope (FIM) for atomic resolution imaging of scanning tunneling microscopy (STM) tips. Work with the scanning electron microscopy (SEM) metrology project for the development of nanotips as SEM field emitters.

- Work with SEMATECH for the development of photolithographically patterned three-dimensional doubleetched structures in silicon.

- Develop methods for the direct interferometry measurements of surface atom spacings with an unbroken uncertainty chain.

- Room temperature chemical preparation of atomically ordered Si surfaces for atom counting and nanofabrication.

Additional information:

The project objective is to meet the future measurement and standards needs of the evolving nanomanufacturing industry. This industry will become pervasive with very difficult but essential standards and interoperability requirements. Whether tooling a new manufacturing facility or making procurement comparisons, accurate tool measures, calibrated standards, and performance evaluation methods will be needed. One challenge facing NIST is the non-availability of standard artifacts or the means to commercially produce them. We are developing, therefore, a parallel effort to fabricate these artifacts and enable their transfer to other metrology tools.

\section{Selected publications}

1. H. Zhou, J. Fu, S. Gonda and R. M. Silver "Effects of Etching Time and Wafer Miscut on the Morphology of Etched Si(111) Surfaces," SPIE Nanotechnology Proc., 2001 (in print).

2. S. Gonda, H. Zhou, J. Fu and R. M. Silver, "A new design and uncertainty consideration of a metrology UHVSTM for direct measurements of atom spacings," SPIE Nanotechnology Workshop Proc., 2001 (in print).

Alignment with NSET Grand Challenges:

\begin{tabular}{|l|l|l|l|l|l|l|l|l|l|l|}
\hline MAT & ELEC & HEALTH & ENV & ENER & SPACE & BIOSEN & TRANS & SECUR & MET & MAN \\
\hline & $\mathrm{X}$ & & & & & & & & $\mathrm{X}$ & \\
\hline
\end{tabular}




\section{Manufacturing Engineering Laboratory}

\section{Optical Manipulation for Testing and Assembly of Micro/Nanoscale Devices}

\section{Project Leader/contact:}

Thomas LeBrun, 301-975-4256, thomas.lebrun@nist.gov

Precision Engineering Division, MEL, Gaithersburg

Approximate staffing:

3.7 FTE

Funding sources

NIST $(100 \%)$

Objective:

To use focused laser beams to manipulate nanometer and $\mu \mathrm{m}$-scale components both in air and in aqueous environments suitable for bio-devices. In this way test nanodevices, assemble them, and measure forces of interaction between components to identify and address the fundamental measurement and standards issues related to fabrication of micro/nanoscale devices. Ultimately these tools will enable testing and assembly of nanodevices, operating in parallel and with noninterfering probes.

\section{Constituency:}

Biotechnology industry, nanomanufacturing industry, and instrument manufacturers.

\section{Principal current tasks:}

- Dither optical trap to create trap potentials shaped to grasp complex components

- Interface trap to virtual reality environment for heuristic manipulation of trapped components

\section{Additional information:}

One of the most pressing requirements for the development of nanotechnology is novel tools to measure and manipulate specimens at the nanometer to micrometer scale - a key point that has been made repeatedly in reports and workshops of the National Nanotechnology Initiative. This project will create new tools to measure the performance of nanodevices, manipulate nanocomponents and provide traceability for force measurements using optical tweezers.

Alignment with NSET Grand Challenges:

\begin{tabular}{|c|c|c|c|c|c|c|c|c|c|c|}
\hline MAT & ELEC & HEALTH & ENV & ENER & SPACE & BIOSEN & TRANS & SECUR & MET & MAN \\
\hline & & $\mathrm{X}$ & & & & $\mathrm{X}$ & & & $\mathrm{X}$ & $\mathrm{X}$ \\
\hline
\end{tabular}




\section{Manufacturing Engineering Laboratory}

\section{Scanning Electron Microscope (SEM) Length Metrology of Nanometer Structures}

\section{Project Leader/contact:}

- Dr. Andras E. Vladar, 301-975-2399, andras@nist.gov

- Dr. Michael T. Postek, 301-975-2299, postek@nist.gov

Precision Engineering Division, MEL, Gaithersburg

Approximate staffing:

3.0 FTE

Funding Sources:

NIST (75\%), Other Agencies (5\%), ISMT (20\%)

Objective:

Development of traceable standard artifacts, infrastructural methodology for dimensional and instrument performance measurements, and calibration procedures.

\section{Constituency:}

The microelectronics industry, including semiconductor integrated circuits (ISMT), magnetic storage, MEMS and emerging nanotechnology industries.

Principal current tasks:

- Development, certification and issuance of RM 8090 and SRM 2090, SEM low and high accelerating voltage magnification calibration standards.

- Development certification and issuance of RM 8120 and SRM 2120, wafer level, production-relevant linewidth standards.

- Infrastructural development of new metrology techniques for nanometer scale devices.

- Development of performance metrics for SEM instrumentation.

- NIST-wide support in high-resolution imaging.

\section{Additional information:}

SEMs are used in many types of research, development and manufacturing. Beyond the extensive use in the microelectronics industry, which has already dimensional metrology needs with accuracies of a few nanometers, SEMs can be used for imaging, measurement and manufacturing of structures with feature sizes down to a few nanometers. It is essential to develop standard artifacts and methods for dimensional measurements, including calibration procedures and instrument performance measurements. A thorough knowledge of the principles of signal generation and formation in the SEM, 3-dimensional modeling of electron beam-sample interaction, and metrology instrumentation for correct sizing and shape measurement are needed for emerging nanotechnology applications.

\section{Selected publications:}

1. Vladar, A. E., Postek, M. T., Zhang, N-F, Larrabee R. D., and Jones, S. N., "Reference Material 8091: new scanning electron microscope sharpness standard," SPIE 4344, 827-834, 2001.

2. Postek, M. T., Vladár, A. E., Wells, O. C., and Lowney, J. L., "Application of the Low-Loss Scanning Electron Microscope (SEM) Image to Integrated Circuit Technology; Part 1, Applications to Accurate Dimension Measurements," SCANNING, p. 289-304, 2001.

Alignment with NSET Grand Challenges:

\begin{tabular}{|c|c|c|c|c|c|c|c|c|c|c|}
\hline MAT & ELEC & HEALTH & ENV & ENER & SPACE & BIOSEN & TRANS & SECUR & MET & MAN \\
\hline $\mathrm{X}$ & $\mathrm{X}$ & & & & & & & & $\mathrm{X}$ & \\
\hline
\end{tabular}




\section{Manufacturing Engineering Laboratory}

\section{Optical Overlay and Linewidth Measurement Methods and Nanometer-scale Standards}

\section{Project Leader/contact:}

Rick Silver, 301-975-5609, richard.silver@nist.gov

Precision Engineering Division, MEL, Gaithersburg

Approximate staffing:

6.0 FTE

Funding sources

NIST (90\%), International SEMATECH (10\%)

Objective:

Provide technological leadership to the emerging nanotechnology and related manufacturing industries by developing and evaluating the methods, tools, and artifacts needed to apply optical techniques to the metrology needs of these industries.

Constituency:

Semiconductor electronics, magnetic storage, MEMS, and emerging nanotechnology industries.

Principal current tasks:

- Continuous development of the NIST overlay metrology tool, measurement methods to obtain uncertainties comparable or better than the best industry overlay tools, and standards to support and calibrate these tools.

- Calibration of NIST Photomask Linewidth Standard SRM 2059, and the development of calibration methods to obtain optical transmission linewidth measurement uncertainties adequate to meet industry needs.

- Development of two-dimensional grid calibration standards, high-accuracy placement standards, and associated measurement techniques, including self-calibration and advanced tool mapping as used by industry.

- Development of leading edge correlation and hardware control algorithms for nanometer scale optical measurements.

Additional information:

Optical methods are used widely in the manufacturing of semiconductor, flat panel, photonic, and storage devices. These industries can be viewed as precursors to the evolving nanomanufacturing industry since they fabricate very small critical dimension structures and have historically pushed metrology to its limit. In this type of leading edge manufacturing, procurement comparisons, accurate tool measures and calibration standards are essential for productivity. The tighter tolerances encountered in the manufacture of nanometer-scale devices place increasing demands on feature linewidth accuracy and on overlay tolerances. Since the parallel nature of high speed, nondestructive optical metrology makes it the tool of choice when applicable, NIST has a comprehensive program to both support and advance the optical techniques needed to make these overlay, pitch, and critical dimension measurements.

\section{Selected publications:}

R. M. Silver, J. Jun, S. Fox, and E. Kornegay, "Recent advances in Overlay Metrology," Future Fab Intl. 11, 2001.

Alignment with NSET Grand Challenges:

\begin{tabular}{|c|c|c|c|c|c|c|c|c|c|c|}
\hline MAT & ELEC & HEALTH & ENV & ENER & SPACE & BIOSEN & TRANS & SECUR & MET & MAN \\
\hline & $\mathrm{X}$ & & & & & & & & $\mathrm{X}$ & \\
\hline
\end{tabular}




\section{Manufacturing Engineering Laboratory}

\section{Scanned Probe Microscope Dimensional Metrology}

Project leader/contact:

- Dr. John Kramar, 301-975-3447, john.kramar@nist.gov

- Dr. Ted Vorburger, 301-975-3493, theodore.vorburger@nist.gov

Precision Engineering Division, MEL, Gaithersburg

Approximate staffing:

4.0 FTE

Funding sources:

NIST $(100 \%)$

Objective:

To provide calibration artifacts and measurement services that support scanned probe microscopy, surface metrology, and dimensional metrology needs of the emerging nanotechnology industry.

\section{Constituency:}

Scanned probe microscope users and nanotechnology industries.

Principal current tasks:

- Provide calibrations and services that support scanned probe microscopy and surface metrology

- Develop metrology instruments for the provision of these measurement capabilities

- Develop artifacts for the dissemination of sub-micrometer step height and pitch traceability

- Support industry's efforts to develop national and international documentary standards in scanned probe microscopy and surface metrology

Additional information:

These measurement capabilities and services are becoming increasingly important both for the development of new nano-scale manufacturing processes and for the continued scale-down of conventional technology. Several artifacts have recently been measured for industry customers. These include measurements of average pitch of standard gratings for the Chandra X-ray Observatory $(200.01 \mathrm{~nm} \pm 10 \mathrm{pm})$, measurements of pitch standards ranging from $200 \mathrm{~nm}$ to $2 \mu \mathrm{m}$, and measurements of step height standards up to $90 \mathrm{~nm}$ in height.

\section{Selected Publications:}

1. R. Dixson, et al., "Silicon Single Atom Steps as AFM Height Standards," Proc. SPIE. 4344, 157 (2001).

2. R. Dixson, et al., "Accurate Dimensional Metrology with Atomic Force Microscopy," Proc. SPIE 3998, 362 (2000).

3. J.A. Kramar, et al., "Grating Pitch Measurements with the Molecular Measuring Machine," Proc. SPIE 3806, $46(1999)$.

4. T.V. Vorburger, et al., "Characterization of Surface Topography," Beam Effects, Surface Topography, and Depth Profiling in Surface Analysis, A. Czanderna et al. eds. (Plenum Press, New York, 1998), p. 275.

Alignment with NSET Grand Challenges:

\begin{tabular}{|c|c|c|l|l|l|l|l|c|c|c|}
\hline MAT & ELEC & HEALTH & ENV & ENER & SPACE & BIOSEN & TRANS & SECUR & MET & MAN \\
\hline & $\mathrm{X}$ & & & & & & & & $\mathrm{X}$ & $\mathrm{X}$ \\
\hline
\end{tabular}




\section{Manufacturing Engineering Laboratory}

\section{Fabrication and Characterization of Nanostructures for Standards}

\section{Project Leader/contact:}

John Dagata, 301-975-3597, john.dagata@nist.gov

Thomas LeBrun, 301-975-4256, thomas.lebrun@nist.gov

Precision Engineering Division, MEL, Gaithersburg

Approximate staffing:

$2.4 \mathrm{FTE}$

Funding sources

NIST $(100 \%)$

Objective:

To develop diverse fabrication techniques for rapid prototyping of functional nanostructures, and apply these techniques to fabricate dimensional and surface texture standards. Fabrication of such standards is being considered in the context of novel self-organizing chemical and biochemical processes that demand chemical functionality as well as structures composed of more traditional metallic and semiconductor materials. Current techniques include scanning probe oxidation, electron-beam lithography, maskless optical lithography, and soft lithography.

Constituency:

Semiconductor electronics industry and nanoscale manufacturing industry.

Principal current tasks:

- Apply scanned probe oxidation (SPO) in tapping mode to fabricate regular arrays of nanostructures with widths of $30 \mathrm{~nm}$ or less.

- Combine SPO with maskless photolithography to pattern large areas with nano-scale features

Additional information:

Few techniques exist for the fabrication of deliberately patterned structures of arbitrary chemical composition that are smaller than 100 nanometers, but such nanoscale structures will be essential for metrological aspects of nanotechnology.

Alignment with NSET Grand Challenges:

\begin{tabular}{|c|c|c|c|c|c|c|c|c|c|c|}
\hline MAT & ELEC & HEALTH & ENV & ENER & SPACE & BIOSEN & TRANS & SECUR & MET & MAN \\
\hline & & & & & & & & & $X$ & $X$ \\
\hline
\end{tabular}




\section{Manufacturing Engineering Laboratory}

\section{Atomic-scale Motion Control for Nanometrology and Nanomanufacturing}

\section{Project Leader/contact:}

Thomas LeBrun, 301-975-4256, thomas.lebrun@nist.gov

Precision Engineering Division, MEL, Gaithersburg

Approximate staffing:

1.9 FTE

Funding sources

NIST $(100 \%)$

\section{Objective:}

Create stages with subnanometer positioning accuracy in multiple degrees of freedom by combining atomicscale motion with picometer interferometry, and real-time servo control. Pair these stages with long-range positioning systems to allow atomic-scale positioning over ranges of $\mathrm{cm}$. Use this technology to provide length measurement services with uncertainties at or below one nanometer.

Constituency:

Microelectronics, optical encoder manufacturers, and nanoscale manufacturers.

Principal current tasks:

- Construct and test second-generation fine stage with subnanometer positioning accuracy

- Test prototype linescale interferometer

\section{Additional information:}

The evolution of nanotechnology is rapidly pushing the limits of our ability to measure length. New tools are also crucial to enable the development of nanotechnology, and for nanomanufacturing this will mean positioning with atomic-scale accuracy over wider ranges than is currently possible. This project is creating stages to provide positioning and motion with subnanometer accuracy to allow nanometrology and nanomanufacturing on macroscopic artifacts (i.e., over ranges of centimeters). This technology is also being applied within NIST to develop a new generation of length measurement services to meet the needs of high-tech industry (microelectronics, optical encoder manufacturers), as well as the needs of the nascent nanotechnology industry.

Alignment with NSET Grand Challenges:

\begin{tabular}{|c|c|c|c|c|c|c|c|c|c|c|}
\hline MAT & ELEC & HEALTH & ENV & ENER & SPACE & BIOSEN & TRANS & SECUR & MET & MAN \\
\hline & & & & & & & & & $X$ & $X$ \\
\hline
\end{tabular}




\section{Manufacturing Engineering Laboratory}

\section{Modeling for Nanometer-Scale Length Metrology}

\section{Project Leader/contact:}

John Villarrubia, 301-975-3958, john.villarrubia@nist.gov

Precision Engineering Division, MEL, Gaithersburg

\section{Approximate staffing:}

2.2 FTE

Funding sources

NIST (75\%), Other (25\%)

\section{Objective:}

Development and improvement of computational models to simulate the geometrical artifacts introduced by measuring instruments, validation of models by appropriate experiments, and inversion of these models (to the extent possible) to deduce the sample geometry that produced a measured image, thereby enabling dimensional metrology with nanometer-scale accuracies and repeatabilities.

\section{Constituency:}

Semiconductor electronics, magnetic storage, MEMS, and emerging nanotechnology industries

Principal current tasks:

- Development of a model-based linewidth and line shape measurement system for the scanning electron microscope (SEM).

- Model predictions of dependence of SEM linewidth measurement repeatability upon measurement conditions such as signal to noise ratio, depth of field, sample geometry, etc.

- Comparisons between experiment and two model codes for measurement of linewidth in optical microscopes.

- Thick layer optical modeling for linewidth and overlay metrology.

\section{Additional information:}

Economically significant existing industries (e.g., semiconductor electronics) already need dimensional metrology with uncertainties of nanometers (see, e.g., the International Technology Roadmap for Semiconductors), and the need is expected to be still more acute for emerging nanotechnology industries, which will produce even smaller structures with more complex geometries. Neither NIST nor industry can perform width or shape measurements to this accuracy without modeling the measurements because interaction between the measuring instrument and the sample produces image distortions on a scale determined by the characteristic size of the probe-sample interaction volume. These probing errors are generally on the scale of some tens of nanometers or larger. Unless understood and corrected, they therefore pose an inherent problem for measurements requiring nanometer-scale accuracy.

\section{Selected publications:}

1. J. S. Villarrubia, A. E. Vladár, J. R. Lowney and M. T. Postek, "Edge Determination for Polycrystalline Silicon Lines on Gate Oxide," Proc. SPIE 4344, 147-156 (2001).

2. J. Potzick, "The problem with submicrometer linewidth standards, and a proposed solution," Proc. SPIE 4344, 139-146 (2001).

Alignment with NSET Grand Challenges:

\begin{tabular}{|c|c|c|c|c|c|c|c|c|c|c|}
\hline MAT & ELEC & HEALTH & ENV & ENER & SPACE & BIOSEN & TRANS & SECUR & MET & MAN \\
\hline & $\mathrm{X}$ & & & & & & & & $\mathrm{X}$ & \\
\hline
\end{tabular}




\section{Manufacturing Engineering Laboratory}

\section{Microforce Realization and Measurement}

\section{Project Leader/contact:}

Jon Pratt, 301-975-5470, jon.pratt@nist.gov

Manufacturing Metrology Division, MEL, Gaithersburg

Approximate staffing:

2.5 FTE

Funding Sources:

NIST (100\%)

Objective:

To establish a metrological basis for small force measurement by developing an electronic realization of force traceable to the SI in the regime between $10^{-8} \mathrm{~N}$ and $10^{-4} \mathrm{~N}$.

\section{Constituency:}

Manufacturers and users of instrumented indentation equipment, manufacturers and users of scanned probe microscopes that are employed in force characterization, and national measurement institutes (NMI's).

Principal current tasks:

- Developing a primary force balance to compare electronic and gravitationally derived forces with the potential to examine atomic and quantum derived forces

- Exploring various small force measurement devices for use as transfer artifacts

- Building a laboratory to house the primary balance

\section{Additional information:}

The measurement of small force is employed to characterize the performance of a variety of industrial products ranging from thin film coatings, such as paints and lacquers, to low thrust propulsion systems for spacecraft, such as field emission electric propulsion. A great deal of scientific interest exists within the biological sciences, where researchers are using small force measurements to unravel the mysteries of protein folding, tissue mechanics, and cellular function. There is also much activity within the physical sciences, where researchers are using small force measurement to confirm the theoretical predictions of quantum mechanics and to probe the physics of the atom. A direct and accurate link to the SI is difficult to achieve for forces less than $10 \mu \mathrm{N}$, owing to difficulties in obtaining and handling small mass artifacts. Recognizing this, we seek to demonstrate the viability of realizing small force using less direct methods based on electronic means. Ultimately, we seek a force balance with a dynamic range that encompasses and can accommodate the smallest available deadweight artifacts, yet is capable of resolving forces on an atomic scale by appealing to the output of a well defined, and metrologically accurate electrostatic force generator.

\section{Selected publications:}

1. D. B. Newell, J.R. Pratt, J.A. Kramar, D.T. Smith, L.A. Feeney, and E. Williams, 2001, "SI Traceability of Force at the Nanonewton Level," Proceedings of the 2001 NCSL International Workshop and Symposium, Washington DC.

2. J. R. Pratt, D.B. Newell, E. R. Williams, D. T. Smith, and John Kramar, 2001, "Towards a Traceable Nanoscale Force Standard", Proceedings of the $2^{\text {nd }}$ EUSPEN International Conference, Turin, Italy, p. 470473.

Alignment with NSET Grand Challenges:

\begin{tabular}{|c|c|c|c|c|c|c|c|c|c|c|}
\hline MAT & ELEC & HEALTH & ENV & ENER & SPACE & BIOSEN & TRANS & SECUR & MET & MAN \\
\hline & & & & & & & & & $X$ & $X$ \\
\hline
\end{tabular}




\section{Manufacturing Engineering Laboratory}

\section{Large Scale Surface Metrology with Sub-Nanometer Uncertainty}

\section{Project Leader/contact:}

- Dr. Ulf Griesmann, 301-975-4929, ulf.griesmann@ nist.gov

- Dr. Tony Schmitz, 301-975-8346, tony.schmitz@nist.gov Manufacturing Metrology Division, MEL, Gaithersburg

Approximate staffing:

2.0 FTE

Funding sources:

NIST (100\%)

\section{Objective:}

Improvement and development of leading-edge optical interferometry of the measurement of surface figures of flat, spheric, conic, and aspheric surfaces with the goal of achieving atomic level uncertainties of $0.25 \mathrm{~nm} \mathrm{rms}$ to meet the needs of optical lithography based industries.

Constituency:

Optics industry, lithography tool industry, semiconductor manufacturing industry, semiconductor material industry.

Principal current tasks:

- Commission the NIST X-ray optics CALIBration InteRferometer (XCALIBIR)

- Develop a flatness standard to meet the need of industry for flatness measurement services and flatness standards.

\section{Additional Information:}

In the future, the manufacture of nanoscale objects with optical lithography will require both advanced optics tools and materials capable of atomic level shape tolerances. XCALIBIR gives us the ability to make these characterizations and thus to provide standards to industry. Established lithography based approaches for manufacturing nanometer scale objects (in chip manufacturing, microelectromechanical systems - MEMS, etc.) have the advantage of massive parallelism over novel "assembler" type approaches. This parallelism is the key to the economic production of nanoscale devices and, therefore, we expect lithography based manufacturing methods to remain critically important.

Alignment with NSET Grand Challenges:

\begin{tabular}{|l|l|l|l|l|l|l|l|l|l|l|}
\hline MAT & ELEC & HEALTH & ENV & ENER & SPACE & BIOSEN & TRANS & SECUR & MET & MAN \\
\hline & $\mathrm{X}$ & & & & & & & & $\mathrm{X}$ & $\mathrm{X}$ \\
\hline
\end{tabular}




\section{Chemical Science and Technology Laboratory}

\section{Molecular Electronics}

\section{Project Leader/contact:}

- Roger D. van Zee, 301-975- 2363, roger.vanzee@nist.gov

Process Measurements Division, CSTL, Gaithersburg

- Curt A. Richter, 301-975-2082, curt.richter@ nist.gov

Semiconductor Electronics Division, EEEL, Gaithersburg

Approximate staffing:

3.25 FTE (CSTL)

Funding sources:

NIST $(100 \%)$

Objective:

To characterize the structural properties and conduction mechanism through molecules and to develop methods to reliably and reproducibly measure the electrical properties of small ensembles of molecules.

Constituency:

Microelectronics industry

Principal current tasks:

- Develop a NIST standard suite of molecular test structures (MTS).

- Develop a fundamental understanding of charge transport through molecules and molecular ensembles.

\section{Additional information:}

This task is part of a cross-disciplinary, inter-laboratory effort at NIST (EEEL and CSTL) with an overall goal of developing the measurement science that will enable molecular electronics (ME) to blossom into a viable industry. Within EEEL, we are developing test-structures based upon nanofabrication and MEMS (Micro-Electro-Mechanical Systems) processing techniques for assessing the electrical properties and reliability of moletronic molecules. In addition to the complexity of the nanofabrication of test structures, the challenges associated with measuring the electrical properties of these small molecular ensembles are daunting. Within CSTL, we are studying the structure of the molecular ensembles inside ME devices and investigating charge transport through these molecules. These experiments are carried out using sophisticated optical spectroscopies and scanned-probe instruments. These molecular systems are being modeled, to provide theoretical insights into the measured transport properties and to validate predictive models.

Alignment with NSET Grand Challenges:

\begin{tabular}{|c|c|c|l|l|l|l|l|c|c|c|}
\hline MAT & ELEC & HEALTH & ENV & ENER & SPACE & BIOSEN & TRANS & SECUR & MET & MAN \\
\hline $\mathrm{X}$ & $\mathrm{X}$ & & & & & & & & $\mathrm{X}$ & \\
\hline
\end{tabular}




\section{Chemical Science and Technology Laboratory}

\section{Self-Assembled Monolayers (SAMs) for Chemical and Biochemical Measurements}

\section{Project Leader/contact:}

Michael J. Tarlov (CSTL), 301-975- 2058, mtarlov@ nist.gov

Process Measurements Division, CSTL, Gaithersburg

Approximate staffing:

2.5 FTE

Funding sources:

NIST $(100 \%)$

Objective:

Establishing a fundamental understanding of the molecular self-assembly processes that govern the formation of monolayers and multi-layers with useful molecular recognition properties.

Constituency:

Biosensing and biotechnology industries

Principal current tasks:

- DNA-functionalized SAMs are being studied as structural elements and molecular-scale "zip-codes" for the assembly of hybrid nano-structures

Additional information:

Measurement methods that provide structural information concerning SAM systems are developed and applied to novel SAM systems to correlate molecular scale structure with sensing and diagnostic behavior. Efforts are primarily concentrated on elucidating the structure of nucleic acid SAMs of the type that are found in DNA chips used for genetic analysis.

Alignment with NSET Grand Challenges:

\begin{tabular}{|l|c|c|c|c|c|c|c|c|c|c|}
\hline MAT & ELEC & HEALTH & ENV & ENER & SPACE & BIOSEN & TRANS & SECUR & MET & MAN \\
\hline & & $\mathrm{X}$ & $\mathrm{X}$ & & & $\mathrm{X}$ & & & $\mathrm{X}$ & \\
\hline
\end{tabular}




\section{Chemical Science and Technology Laboratory}

\section{Combinatorial Methods}

\section{Project Leader/contact:}

Steven A. Buntin, 301-975-2989, steven.buntin@nist.gov

Surface and Microanalysis Science Division, CSTL, Gaithersburg

Approximate staffing:

2.0 FTE

Funding sources:

NIST $(100 \%)$

Objective:

To develop measurement methods that can evaluate smaller and smaller samples, extract meaningful data on nanoscale properties, and provide sample array platforms that can be independently heated and addressed.

Constituency:

Laboratory instrumentation sector, materials performance characterization laboratories

Principal current tasks:

- Develop surface and microanalysis methods that will allow hyperspectral data collection on a multiplexed measurement platform.

- Assess adhesion of polymer blend libraries by high throughput methods using Raman imaging instrumentation.

- Develop a non-contact, hyperspectral scanned probe capable of simultaneous measurement of sample properties using microwave, infrared, visible, and ultraviolet spectroscopies.

Alignment with NSET Grand Challenges:

\begin{tabular}{|l|l|l|l|l|l|l|l|c|c|c|}
\hline MAT & ELEC & HEALTH & ENV & ENER & SPACE & BIOSEN & TRANS & SECUR & MET & MAN \\
\hline & & & & & & & & & $X$ & \\
\hline
\end{tabular}




\section{Chemical Science and Technology Laboratory}

\section{Chemical Nanoanalysis}

\section{Project Leader/contact:}

Eric B. Steel, 301-975-3902, eric.steel@nist.gov

Surface and Microanalysis Science Division, CSTL, Gaithersburg

Approximate staffing:

2.0 FTE

Funding sources:

NIST (50\%), Other Agencies (50\%)

Objective:

To establish nanometer-scale chemical measurements and standards.

Constituency:

Electronics industry, powder technologies, thin film coatings, environmental

Principal current tasks:

- Develop electron and x-ray spectroscopic measurement methods on electron beam and synchrotron instruments to allow high spatial resolution analysis

- Develop and analyze thin film and nanoparticle test materials are being to determine the best chemical nanoanalysis approaches

Alignment with NSET Grand Challenges:

\begin{tabular}{|l|c|l|l|l|l|l|l|c|c|c|}
\hline MAT & ELEC & HEALTH & ENV & ENER & SPACE & BIOSEN & TRANS & SECUR & MET & MAN \\
\hline & $\mathrm{X}$ & & & & & & & & $\mathrm{X}$ & \\
\hline
\end{tabular}




\section{Chemical Science and Technology Laboratory}

\section{Molecular Scale Characterization}

\section{Project Leader/contact:}

Lee J. Richter, 301-975-4152, lee.richter@nist.gov

Surface and Microanalysis Science Division, CSTL, Gaithersburg

Approximate staffing:

3.5 FTE

Funding sources:

NIST $(100 \%)$

Objective:

To develop nonlinear optic methods and Secondary Ion Mass Spectrometry (SIMS) to probe interface structure and dynamics at the molecular level.

Constituency:

Electronics industry, coatings industry

Additional information:

Nonlinear optic methods and Secondary Ion Mass Spectrometry (SIMS) are being developed to probe interface structure and dynamics at the molecular level. The technique of vibrationally-resonant sum frequency generation uniquely provides information concerning molecular bonding specifically at the interface between two media (e.g., solid/solid, solid/liquid, etc.). Methodologies have been developed that led to the characterization of the detailed structure of polystyrene/glass interfaces, including the determination of the alignment of molecular groups along the polymer backbone. Cluster ion source advances in SIMS are being pursued to increase the sensitivity and reduce the degree of surface damage to allow sub-monolayer sensitivity to molecular species. Cluster source methods are currently being applied to several polymer films to assess the general applicability of the method.

Alignment with NSET Grand Challenges:

\begin{tabular}{|l|l|l|l|l|l|l|l|c|c|c|}
\hline MAT & ELEC & HEALTH & ENV & ENER & SPACE & BIOSEN & TRANS & SECUR & MET & MAN \\
\hline & & & & & & & & & $X$ & \\
\hline
\end{tabular}




\section{Chemical Science and Technology Laboratory}

\section{Nearfield Methods for Chemical Analysis}

Project Leader/contact:

Stephan J. Stranick, 301-975-2348

Surface and Microanalysis Science Division, CSTL, Gaithersburg

Approximate staffing:

1.0 FTE

Funding sources:

NIST $(100 \%)$

Objective:

To use near-field optical methods to combine the spatial resolution of scanned probes with the spectral information of optical methods.

Constituency:

Industries utilizing characterization of chemical nanostructures (from catalysts to microelectronics)

Principal current tasks:

- To extend near-field methods to incorporate Raman scattering and infrared absorption to develop measurement methods capable of following chemical changes on the nanoscale.

Additional information:

Recent efforts have focused on accurately assessing sensitivity and resolution for NSOM-based infrared absorption using a broadband femtosecond laser based source, and applying this nanoscale, hyperspectral imaging instrumentation to polymer blend systems.

Alignment with NSET Grand Challenges:

\begin{tabular}{|l|l|l|l|l|l|l|l|c|c|c|}
\hline MAT & ELEC & HEALTH & ENV & ENER & SPACE & BIOSEN & TRANS & SECUR & MET & MAN \\
\hline & & & & & & & & & $X$ & \\
\hline
\end{tabular}




\section{Physics Laboratory}

\section{Nanoscale Physics Research}

\section{Project Leader/contact:}

Robert Celotta, 301-975-3710, robert.celotta@nist.gov

Electron and Optical Physics Division, PL, Gaithersburg

Approximate staffing:

8 FTE

Funding sources:

NIST (91\%), Other Agencies (9\%)

\section{Objective:}

To perform research in nanostructure measurement science, including electron-surface interactions, surface and multilayer magnetism, laser-focused atom deposition, electron interaction theory, electron spin polarization phenomena, and electron optics and instrumentation.

Constituency:

Magnetics industry, particularly magnetic storage in information technology; electronics industry; information technology, quantum computing; materials industry

Principal current tasks:

- Magnetic imaging of magnetic nanostructures using a new scanning electron microscope (SEM) with polarization analysis (SEMPA)

- Development of spin polarized scanning tunneling microscopy (STM)

- Single atom manipulation under optical control

Additional information:

The immediate goal of the SEMPA work is to achieve a spatial resolution of $\approx 10 \mathrm{~nm}$ for magnetic imaging. The STM effort is directed toward using polarized photons from a GaAs tip to determine the domain structure of a magnetic nanostructure in an STM with very high spatial resolution. Finally, the atom manipulation work has the immediate goal of producing single atoms on demand.

Selected publications:

http://physics.nist.gov/Divisions/Div841/Gp3/epg_files/pub.html

Alignment with NSET Grand Challenges:

\begin{tabular}{|c|c|c|c|c|c|c|c|c|c|c|}
\hline MAT & ELEC & HEALTH & ENV & ENER & SPACE & BIOSEN & TRANS & SECUR & MET & MAN \\
\hline $\mathrm{X}$ & $\mathrm{X}$ & & & & & & & & $\mathrm{X}$ & \\
\hline
\end{tabular}




\section{Physics Laboratory}

\section{Nanoelectronics and Autonomous Atom Assembly}

\section{Project Leader/contact:}

Robert Celotta, 301-975-3710, robert.celotta@nist.gov

Electron and Optical Physics Division, PL, Gaithersburg

Approximate staffing:

4 FTE

Funding sources:

NIST (84\%), Other Agencies (16\%)

Objective:

To establish the fundamental physical principles of importance in electronic nanostructures through the atomic scale investigation of quantum electronics.

Constituency:

Electronics, magnetics, and materials industries

Principal current tasks:

- To fabricate electronic and magnetic nanostructures and measure their physical properties with atomic scale resolution

\section{Additional information:}

The laboratory came on-line with the successful operation of the main measurement system, a scanning tunneling microscope (STM) operating at cryogenic temperatures in an intense magnetic field. The cryogenic STM was designed and built at NIST. Novel features of the microscope include a solid molybdenum body design (made possible by the high-speed machining capabilities at NIST), an integral 3-axes positioning system of the sample and tip with picometer precision, optical access to the sample/tip junction, and non-magnetic construction. The microscope is a completely self-contained unit that can be translated between a room temperature system and a liquid $\mathrm{He}$ cryostat. The microscope was designed to have a very high spatial and energy resolution. It can measure displacements below $1 \mathrm{pm}$ and resolve electron energy levels separated by $600 \mu \mathrm{V}$. Operating in a cryogenic, high magnetic field and ultra-high vacuum environments the microscope measures electronic and magnetic properties of nanostructures on an atom-by-atom basis. The microscope has achieved atomic resolution measurements on the surface of $\mathrm{Cu}(111)$ at $2.3 \mathrm{~K}$ and in magnetic fields up to $10 \mathrm{~T}$. The stability of the microscope allows atomic resolution measurements to remain in registry while the magnetic field is swept. The laboratory contains facilities for the fabrication of samples, tips, and nanostructures. The facilities include traditional molecular-beam epitaxy of III-V semiconductors, superconductors, and magnetic materials, as well as bottom-up nanofabrication using autonomous atom assembly.

Selected publications:

http://physics.nist.gov/Divisions/Div841/Gp3/epg_files/pub.html

Alignment with NSET Grand Challenges:

\begin{tabular}{|c|c|c|c|c|c|c|c|c|c|c|}
\hline MAT & ELEC & HEALTH & ENV & ENER & SPACE & BIOSEN & TRANS & SECUR & MET & MAN \\
\hline $\mathrm{X}$ & $\mathrm{X}$ & & & & & & & & $\mathrm{X}$ & $\mathrm{X}$ \\
\hline
\end{tabular}




\section{Physics Laboratory}

\section{Laser Trapping and Cooling}

\section{Project Leader/contact:}

Bill Phillips, 301-975-6554, william.phillips@nist.gov

Atomic Physics Division, PL, Gaithersburg

Approximate staffing:

4 FTE

Funding sources:

NIST $(100 \%)$

Objective:

To study the physics of laser cooling, electromagnetic trapping, and other radiative manipulation of neutral atoms and dielectric particles.

Constituency:

Biotechnology and microelectronics industries.

Principal current tasks:

- To develop techniques using optical tweezers, e.g., to measure stochastically-driven on and off rates of adhesion to biomolecules immobilized on surfaces.

- $\quad$ To perform research in coherent atom optics -- atoms released from a Bose-Einstein Condensate.

Additional information:

These fundamental studies are used to develop applications to new kinds of physics measurements and processes such as high resolution spectroscopy, atomic clocks, atomic collisions, atom optics, bio-molecular interactions, and atomic-scale and nano-scale fabrication. The group is lead by Bill Phillips (Nobel Prize in Physics 1997).

We have measured the distribution of times for formation and dissociation of antigen-antibody bonds and initiated theoretical modeling of the experimental results. We have constructed a new apparatus incorporating both optical tweezers and an "optical scalpel" to cut into biological objects. We have initiated studies of liposome manipulation using both the optical tweezers and scalpel.

Using coherent atom optics, we have demonstrated non-linear effects with these nanoscale beams, such as four-wave mixing, which increases the potential for eventual application of these beams in the control of atomic deposition on the nanometer scale.

Selected publications:

http://physics.nist.gov/Divisions/Div842/Gp4/group4.html

Alignment with NSET Grand Challenges:

\begin{tabular}{|c|c|c|l|l|l|l|l|c|c|c|}
\hline MAT & ELEC & HEALTH & ENV & ENER & SPACE & BIOSEN & TRANS & SECUR & MET & MAN \\
\hline $\mathrm{X}$ & & $\mathrm{X}$ & & & & & & & $\mathrm{X}$ & $\mathrm{X}$ \\
\hline
\end{tabular}




\section{Physics Laboratory}

\section{Atomic Radiation and Plasma}

\section{Project Leader/contact:}

Wolfgang Wiese, 301-975-3201,wolfgang.wiese@ nist.gov

Atomic Physics Division, PL, Gaithersburg

Approximate staffing:

1.7 FTE

Funding sources:

NIST $(100 \%)$

Objective:

To perform experimental and theoretical research on quantum processes in atomic, molecular, and nanoscale systems, including exploration of atomic interactions in plasmas and with surfaces.

Constituency:

Microelectronics industry and quantum computing

Principal current tasks:

- The NIST electron beam ion trap (EBIT)

- Fundamental measurements on reference plasmas to assist industry in diagnosing industrially-important plasmas, such as those used in micro- and nano-scale etching processes

\section{Additional information:}

The EBIT is used to make exotic, highly charged ions that carry large amounts of potential energy. The ions in these beams interact with surfaces to create nanoscale features that are studied using scanning tunneling microscopy (STM) and atomic force microscopy (AFM) techniques.

This project also includes the precision measurement of the optical properties of materials proposed for inclusion in $193 \mathrm{~nm}$ and $157 \mathrm{~nm}$ optical lithography systems. These developmental activities support the integrated circuit (semiconductor) industry in their target of achieving feature sizes on the order of $70 \mathrm{~nm}$. NIST recently made the significant discovery that calcium fluoride, one of the few materials that are transparent at $157 \mathrm{~nm}$, is inherently birefringent in the deep ultraviolet. The practical import for optical engineers is that a calcium fluoride lens will not focus properly without, at the very least, more careful control of polarization than has been previously required. This is a difficult problem given that there are about 20 lens elements in a typical wafer stepper. NIST researchers are also pursuing potential solutions to the problem involving mixed crystals to compensate for the birefringence effect.

Selected publications:

http://physics.nist.gov/Divisions/Div842/Gp3/group3.html

Alignment with NSET Grand Challenges:

\begin{tabular}{|c|c|c|l|l|l|l|l|c|c|c|}
\hline MAT & ELEC & HEALTH & ENV & ENER & SPACE & BIOSEN & TRANS & SECUR & MET & MAN \\
\hline $\mathrm{X}$ & $\mathrm{X}$ & & & & & & & & $\mathrm{X}$ & $\mathrm{X}$ \\
\hline
\end{tabular}




\section{Physics Laboratory}

\section{Quantum Information Competence}

\section{Project Leader/contact:}

Carl Williams, 301-975-3531, carl.williams@ nist.gov

Atomic Physics Division, PL, Gaithersburg

Approximate staffing:

10.6 FTE

Funding sources:

NIST (58\%), Other Agencies (42\%)

Objective:

To develop qubit systems based on nanoscale systems of atoms and ions for Quantum Information.

Constituency:

Quantum computing, data encryption

Principal current tasks:

- To eliminate and/or overcome the effects of motional heating (which degrades the fidelity of the data-bus qubit)

- To multiplex ion-trap systems by transferring ions (i.e., quantum information) between processor arrays

- Tests possible implementations of quantum logic operations in optically confined systems

- Uniform loading of optical lattice sites with a predetermined number of atoms

- Addressing of the individual qubits in an optical lattice

- Entanglement of atoms at lattice sites

- Providing detailed modeling of current experimental systems, on identifying fundamental limitations due to effects of noise and decoherence

- Evaluating alternative approaches for implementing quantum logic in trapped-atom systems

Additional information:

Quantum Information touches some the most fundamental questions of modern physics, and there are long-term benefits to NIST as the subject bears on fundamental questions of metrology and time keeping. But it also has practical applications of interest in communications, cryptography, and complexity theory. NIST has the most capable programs in the most promising approaches to Quantum Information, which involve nanoscale systems of atoms and ions.

Selected publications:

http://qubit.nist.gov/index.html

Alignment with NSET Grand Challenges:

\begin{tabular}{|c|c|c|c|c|l|l|l|c|c|c|}
\hline MAT & ELEC & HEALTH & ENV & ENER & SPACE & BIOSEN & TRANS & SECUR & MET & MAN \\
\hline $\mathrm{X}$ & $\mathrm{X}$ & & & & & & & $\mathrm{X}$ & $\mathrm{X}$ & \\
\hline
\end{tabular}




\section{Physics Laboratory}

\section{Bose Condensation Experiments}

\section{Project Leader/contact:}

Eric Cornell, 303-492-6281, cornell@jila.colorado.edu

Quantum Physics Division, PL, Boulder

Approximate staffing:

0.9 FTE

Funding sources:

NIST $(100 \%)$

Objective:

Explore and exploit the ultimate limits in low-temperature gases - including both fermions and bosons.

Constituency:

Quantum computing.

Principal current tasks:

- Probe the decay of so-called "internal" coherence of the Bose condensate, the relative quantum phase between the nuclear spin-states of the component atoms

- Perform experiments on Bosenovas to gain insight into the nature of shock waves in these systems

\section{Additional information:}

In 1995, NIST physicists achieved a temperature far lower than has ever been produced before and created an entirely new state of matter predicted decades ago by Albert Einstein and Indian physicist Satyendra Nath Bose -called a Bose-Einstein condensate (BEC). In a BEC, the quantum mechanical profiles of the atoms spread out and overlap, causing the individual atoms to condense into a "superatom" behaving as a single entity.

Today, this project explores and exploits the ultimate limits in low-temperature gases. The two basic species of atoms -- fermions and bosons -- behave very differently at ultra-low temperatures. Each offers opportunities in precision sensing and metrology, and in each case the effects of quantum mechanics, ordinarily masked by random thermal behavior, are dominant.

Selected publications:

http://www.bec.nist.gov

Alignment with NSET Grand Challenges

\begin{tabular}{|c|l|l|l|l|l|l|l|c|c|c|}
\hline MAT & ELEC & HEALTH & ENV & ENER & SPACE & BIOSEN & TRANS & SECUR & MET & MAN \\
\hline $\mathrm{X}$ & & & & & & & & & $X$ & \\
\hline
\end{tabular}




\section{Materials Science and Engineering Laboratory}

\section{Nanotribology}

\section{Project Leader/contact:}

Stephen M. Hsu, 301-975-6120, stephen.hsu@nist.gov

Ceramics Division, MSEL, Gaithersburg

Approximate staffing:

5.0 FTE

Funding sources:

NIST (80\%), Other Agencies (20\%)

\section{Objective:}

To provide measurement methods, instrumentation, and critical data on friction, stiction, and adhesion on relevant materials to the user community through workshops, publications, presentations, and collaborative research networks.

Constituency:

Manufacturers and users of magnetic hard disks, sensors, actuators, microelectromechanical systems (MEMS), biomedical devices, and integrated microsystems.

Principal current tasks:

- Monomolecular films to control the surface properties of device materials

- Design of molecular assembly to control the surface properties of materials

- Scaling laws relating $\mathrm{nm}$ material properties measurements to $\mathrm{mm}$ measurements

- Accurate friction measurement on engineering surfaces

- Test method development to measure durability of carbon overcoats and lubricant combination under a high speed impact condition on magnetic hard disks

\section{Additional information:}

Small scale components fail due to friction and adhesion. Durability is a chief industrial concern for MEMs, actuators, and microsystems. At the nano-scale, surface forces, electrostatic forces, and long range forces become significant in addition to mechanical forces. Nano-friction is the sum of friction + adhesion (a function of contact area). Overcoming and controlling friction at the nanoscale will enhance sense-compute-actuate processes in real time; enable new devices that require contact motion to operate; and provide long term durability criteria for such devices

Selected publications:

1. "Scaling Issues in the Measurement of Monolayer Films," S. M. Hsu, P. M. McGuiggan, J. Zhang, Y. Wang, F. Yin, Y. P. Yeh, and R. S. Gates, in 'Micro- Nano-Tribology' NATO ASI series, edited by Bharat Bhushan, Kluwer Academic Publishers, Netherlands, p.691-710, 2001

2. "Friction Measurements of Ultra-Thin Carbon Overcoats in Air," P. M. McGuiggan, S.M. Hsu, W. Fong, D. Bogy, and C. S. Bhatia, J. of Tribology, vol. 123, 1-6, 2001.

Alignment with NSET Grand Challenges:

\begin{tabular}{|c|c|c|c|c|c|c|c|c|c|c|}
\hline MAT & ELEC & HEALTH & ENV & ENER & SPACE & BIOSEN & TRANS & SECUR & MET & MAN \\
\hline $\mathrm{X}$ & $\mathrm{X}$ & & & & $\mathrm{X}$ & & & & $\mathrm{X}$ & \\
\hline
\end{tabular}




\section{Materials Science and Engineering Laboratory}

\section{Electromigration in Interconnects}

Project Leader/contact:

Robert Keller, 303-497-3853, keller@boulder.nist.gov

Materials Reliability Division, MSEL, Boulder

Approximate staffing:

$1.5 \mathrm{FTE}$

Funding sources:

NIST $(100 \%)$

Objective:

This project addresses the mechanisms that control reliability in chip-level interconnections. Specific activities include studies of time-varying reliability degradation phenomena, localized stress measurement, and understanding the role of local variations in microstructure in electromigration and thermal stress-induced failure.

Constituency:

Semiconductor electronics industry

Principal current tasks:

- Investigate new reliability-limiting phenomena in nanometer-scale interconnections

- Develop the measurement methods required to understand in detail the entire distribution of microstructure

\section{Additional information:}

The project addresses reliability-controlling mechanisms and the resulting properties which, in turn, will allow construction of accurate design models for new generations of microelectronic and optoelectronic structures. This work has identified a new failure mechanism for thin film metallic interconnects in microelectronic devices. Division staff, in collaboration with the Max-Planck-Institute for Metals Research, have identified the presence of surface damage in aluminum alloy and copper interconnects that were stressed by application of an AC electrical current. The damage is consistent with that observed due to fatigue in bulk structural metals. Cyclic deformation of such interconnects can occur during normal use and presents a potentially serious reliability threat to performance of devices incorporating soft polymeric dielectric layers.

\section{Selected publications:}

Keller, R.R., Mönig, R., Volkert, C.A., Arzt, E., Schwaiger, R., and Kraft, O., "Interconnect Failure due to Cyclic Loading," in Stress-Induced Phenomena in Metallizations: Sixth International Workshop, American Institute of Physics, in press (2002).

Alignment with NSET Grand Challenges:

\begin{tabular}{|l|c|l|l|l|l|l|l|c|c|c|}
\hline MAT & ELEC & HEALTH & ENV & ENER & SPACE & BIOSEN & TRANS & SECUR & MET & MAN \\
\hline & $\mathrm{X}$ & & & & & & & & $\mathrm{X}$ & \\
\hline
\end{tabular}




\section{Materials Science and Engineering Laboratory}

\section{Atomic Force Acoustic Microscopy (AFAM)}

\section{Project Leader/contact:}

Donna Hurley, 303-497-3081, hurley@boulder.nist.gov

Materials Reliability Division, MSEL, Boulder

Approximate staffing:

1.0 FTE

Funding sources:

NIST $(100 \%)$

Objective:

Develop in-situ, nondestructive measurement techniques to probe mechanical properties on the nanoscale.

\section{Constituency:}

Microelectronics industry, nanoscale manufacturers, optoelectronics industry, magnetic recording industry, biomedical industry.

Principal current tasks:

- Adapt experimental and analysis techniques for improved measurements (softer materials, different cantilevers, etc.)

- Improve software for rapid, quantitative images and apply to samples with mechanical inhomogeneity

\section{Additional information:}

To cope with ever-decreasing length scales, development of new materials measurement tools is critical. One such need is a method to determine mechanical properties with submicrometer resolution. Methods must be able to provide information in image form to better understand complex structures, as well as provide quantitative results on materials ranging from soft tissue or polymers to hard metals or silicon. AFAM involves acoustically driving the atomic force microscopy (AFM) cantilever to excite its mechanical resonances when it is in contact with a sample. AFAM could prove useful for nondestructively evaluating materials or fabricated structures with variations in mechanical properties on the nanoscale. Qualitative images of relative elasticity have been obtained by measuring the cantilever's vibration amplitude as the tip was scanned across the sample at a fixed excitation frequency.

Quantitative AFAM information has been obtained by analyzing shifts in the resonant frequencies. Finite element models are being developed through a university collaboration to quantify the AFAM response of cantilevers with nonuniform shape and to probe the complex tip-sample interaction.

\section{Selected publications:}

D. C. Hurley, J. S. Wiehn, J. A. Turner, and P. S. Rice, "Quantitative elastic-property information with acoustic AFM: measurements and modeling," conference proceedings of the SPIE Symposium on NDE and Reliability of Micro- and Nanosystems (17-20 March 2002, San Diego, CA).

Alignment with NSET Grand Challenges:

\begin{tabular}{|c|c|c|c|c|c|c|c|c|c|c|}
\hline MAT & ELEC & HEALTH & ENV & ENER & SPACE & BIOSEN & TRANS & SECUR & MET & MAN \\
\hline & $\mathrm{X}$ & $\mathrm{X}$ & & & & & & & $\mathrm{X}$ & \\
\hline
\end{tabular}




\section{Materials Science and Engineering Laboratory}

\section{Mechanical Behavior of Thin Films}

\section{Project Leader/contact:}

Dave Read, 303-497-3853, read@boulder.nist.gov

Materials Reliability Division, MSEL, Boulder

Approximate staffing:

2.0 FTE

Funding sources:

NIST $(100 \%)$

Objective:

Develop measurement techniques for mechanical behavior of micro- and nanoscale thin films.

\section{Constituency:}

Semiconductor electronics industry, microelectronics packaging industry

\section{Principal current tasks:}

- Extend test techniques from their present level (1 $\mu \mathrm{m}$ thick, $10 \mu \mathrm{m}$ wide) to smaller specimens that are similar in size to the conductive traces used in contemporary VLSI (very large scale integration) circuits (widths of $0.1 \mu \mathrm{m}$ to $1 \mu \mathrm{m}$ ).

\section{Additional information:}

Thin films, ubiquitous in microelectronics and photonics, are formed by a wide variety of advanced fabrication techniques. Their microstructures and mechanical properties are different from those of the bulk material, if indeed the material actually exists in bulk form. The general principles of conventional mechanical testing are applicable to thin films, but special equipment and techniques are required. Furthermore, in order to utilize sophisticated mechanical design approaches for new micro- and nanoscale structures and devices, measurement techniques suitable for the materials that take proper account of their size and connectivity to the overall structures, are needed. This year we successfully applied the technique to measure two new types of materials: aluminum interconnect layers made in a commercial CMOS (complementary metal oxide silicon) fabrication facility, obtained through the MOSIS service, and polycrystalline silicon made at Sandia National Laboratories.

\section{Selected publications:}

Read, D.T., Cheng, Y.-W., Keller, R.R., and McColskey, J.D., "Tensile Properties of Free-Standing Aluminum Thin Films," Scripta Mater. 45, 583 (2001).

Alignment with NSET Grand Challenges:

\begin{tabular}{|c|c|c|c|c|c|c|c|c|c|c|}
\hline MAT & ELEC & HEALTH & ENV & ENER & SPACE & BIOSEN & TRANS & SECUR & MET & MAN \\
\hline & $\mathrm{X}$ & & & & & & & & $\mathrm{X}$ & \\
\hline
\end{tabular}




\section{Materials Science and Engineering Laboratory}

\section{Packaging Reliability}

\section{Project Leader/contact:}

Andrew Slifka, 303-497-3744, slifka@boulder.nist.gov

Materials Reliability Division, MSEL, Boulder

Approximate staffing:

1.9 FTE

Funding sources:

NIST $(100 \%)$

Objective:

Develop methods to examine materials and interfaces in electronic packaging and elucidate damage mechanisms.

Constituency:

Microelectronics packaging industry

Principal current tasks:

- Measure mechanical strain in advanced packaging structures and embedded passive materials using electron-beam moiré.

- Measure heat flow and thermal properties on increasingly smaller size scales using thermal infrared and atomic force microscopy (AFM)-nanoprobe microscopy.

\section{Additional information:}

Increased packaging densities in the microelectronics and other nanotechnology-related industries can lead to increased thermomechanical failures. The necessary measurement methods for thermal transport through package structures and for interfacial strain and resulting failure on the nanoscale are being developed in this project. In the short term, industry will be provided with design verification measurements and in the long term with measurement systems.

Selected publications:

Drexler, E.S., "Plastic Strain in Thermally Cycled Flip-Chip PBGA Solder Bump," IEEE Trans. Adv.

Packaging 23, 646 (2000).

Alignment with NSET Grand Challenges:

\begin{tabular}{|c|c|c|c|c|c|c|c|c|c|c|}
\hline MAT & ELEC & HEALTH & ENV & ENER & SPACE & BIOSEN & TRANS & SECUR & MET & MAN \\
\hline & $\mathrm{X}$ & & & & & & & & $\mathrm{X}$ & \\
\hline
\end{tabular}




\section{Materials Science and Engineering Laboratory}

\section{Brillouin Light Scattering for Thin Film Analysis}

\section{Project Leader/contact:}

Ward Johnson, 303-497-5805, wjohnson@boulder.nist.gov

Materials Reliability Division, MSEL, Boulder

Approximate staffing:

$0.8 \mathrm{FTE}$

Funding sources:

$$
\text { NIST (100\%) }
$$

\section{Objective:}

Develop and apply methods for measuring the dynamic elastic and magnetic properties of thin films at gigahertz frequencies with micron-scale resolution lateral resolution, enabling the determination of elastic and dynamic magnetic properties of films as thin as a few nanometers.

\section{Constituency:}

Magnetic recording, optoelectronics, and microelectronics industries.

Principal current tasks:

- Construct the Brillouin light scattering apparatus.

\section{Additional information:}

Brillouin light scattering is a technique to characterize both phonons and magnons in thin-film materials. Because of this dual capability, it can provide unique information on dynamic magnetoacoustic coupling, which is critical to the performance of magnetic recording devices. The sensitivity to mid-gigahertz modes, combined with the fact that lateral excitation areas are limited only by the laser spot size, provides spatial resolutions that are unmatched by conventional ultrasonic methods and presents numerous possibilities for measurements relating to the performance and reliability of optoelectronic and microelectronic thin-film devices. The high frequencies enable the characterization of spatial variations of properties within a thin film and, thus, can provide information relating to adhesion, mechanical surface damage, and chemical surface contamination. The short wavelengths also present the possibility of obtaining detailed information on performance-limiting interactions within patterned thin-film structures, such as magnetic and quantum dots.

Alignment with NSET Grand Challenges:

\begin{tabular}{|c|c|c|l|l|l|l|l|c|c|c|}
\hline MAT & ELEC & HEALTH & ENV & ENER & SPACE & BIOSEN & TRANS & SECUR & MET & MAN \\
\hline & $\mathrm{X}$ & & & & & & & & $\mathrm{X}$ & \\
\hline
\end{tabular}




\section{Materials Science and Engineering Laboratory}

\section{Mechanical Properties of Thin Films using Acoustic Waves}

\section{Project Leader/contact:}

Vinod Tewary, 303-497-5753, tewary@boulder.nist.gov

Materials Reliability Division, MSEL, Boulder

Approximate staffing:

$1.5 \mathrm{FTE}$

Funding sources:

NIST $(100 \%)$

Objective:

Develop and apply theoretical and experimental acoustic methods to determine the elastic properties of advanced anisotropic materials.

Constituency:

Semiconductor electronics industry, wear and hardness coating manufacturers, advanced materials manufacturers

Principal current tasks:

- Apply methods to new material systems (e.g., ultra-low-k dielectrics, nanocomposites).

- Complete elastodynamic Green's function model for two thin anisotropic films on a semiconductor single crystal substrate. Apply to surface acoustic wave (SAW) measurement to obtain film properties.

- Calculate elastostatic and lattice-static Green's functions, including stresses and strains from a static point load or multiscale modeling of point defects in films or film-substrate interfaces.

- Participate in an international comparison between SAW and instrumented indentation testing (IIT) data.

\section{Additional information:}

Acoustic wave propagation is a powerful tool for nondestructively characterizing thin films critical to such applications as solid-state devices, hardness coatings, or diffusion barriers. With this method, information about properties like Young's modulus can be obtained on as-deposited films to provide process information or performance feedback. The experimental approach is based on the optical generation and detection of highfrequency surface acoustic waves. A key component is the development of analytical models based on the elastodynamic Green's functions to model the propagation of the waves in anisotropic films. This project also has an extensive outreach effort in which the models are made available through a web-based project involving education institutions and industrial modeling laboratories.

Selected publications:

1. V. K. Tewary, "Elastic wave propagation in an anisotropic film on an anisotropic substrate: Theory and application to titanium nitride films on single-crystal silicon," submitted to J. Acoust. Soc. Am. (2001).

2. D. C. Hurley, V. K. Tewary, and A. J. Richards, "Surface acoustic wave methods to determine the anisotropic elastic properties of thin films," Meas. Sci. Technol. 12, 1486 (2001).

3. J. R. Berger and V. K. Tewary, "Green's functions for boundary element analysis of anisotropic bimaterials," Engineering Analysis with Boundary Elements 25, 279 (2001).

Alignment with NSET Grand Challenges:

\begin{tabular}{|c|c|c|l|l|l|l|l|c|c|c|}
\hline MAT & ELEC & HEALTH & ENV & ENER & SPACE & BIOSEN & TRANS & SECUR & MET & MAN \\
\hline & $\mathrm{X}$ & & & & & & & & $\mathrm{X}$ & \\
\hline
\end{tabular}




\section{Materials Science and Engineering Laboratory}

\section{Characterization of Porous Low-k Dielectric Constant Thin Films}

\section{Project Leader/contact:}

Barry J. Bauer, 301-975-6849, barry.bauer@nist.gov

Polymers Division, MSEL, Gaithersburg

Approximate staffing:

3.3 FTE

Funding sources:

NIST (64\%), Other (36\%)

Objective:

To develop a lab-based metrology for measuring thin film pore size distributions. Provide structure and property information (composition, porosity, coefficient of thermal expansion - CTE, average pore size, moisture uptake, pore connectivity) of nanoporous low-k thin films to International Sematech, material suppliers and other microelectronic companies.

Constituency:

Semiconductor electronics industry, nanoscale manufacturers

Principal current tasks:

- Characterization of current industrially relevant materials submitted by International SEMATECH

- Developing advanced measurement methods for more thorough and accurate film characterization

\section{Additional information:}

The methodology utilizing several complementary experimental techniques to measure the average pore and matrix morphology has been expanded to include pore size distributions. New methods have been developed to measure pore and matrix morphology in films having complex structures with any morphological type.

Selected publications:

"Properties of nanoporous silica thin films determined by high-resolution x-ray reflectivity and small-angle neutron scattering” W. L. Wu, W. E. Wallace, E. K. Lin, G. W. Lynn, C. J. Glinka, E. T. Ryan and H. M. Ho, J. Appl. Phys., 87, 1193 (2000).

Alignment with NSET Grand Challenges:

\begin{tabular}{|c|c|c|c|c|c|c|c|c|c|c|}
\hline MAT & ELEC & HEALTH & ENV & ENER & SPACE & BIOSEN & TRANS & SECUR & MET & MAN \\
\hline & $\mathrm{X}$ & & & & & & & & $\mathrm{X}$ & \\
\hline
\end{tabular}




\section{Materials Science and Engineering Laboratory}

\section{Fluorescent Sub-Micron Probe Molecules with Starlike Architecture}

\section{Project Leader/contact:}

Barry J. Bauer, 301-975-6849, barry.bauer@nist.gov

Polymers Division, MSEL, Gaithersburg

Approximate staffing:

1.0 FTE

Funding sources:

NIST $(100 \%)$

Objective:

To develop fluorescent polymeric markers for use as probes of sub-micron features in membranes, polymer blends, and biomaterials.

Constituency:

Biomaterials industry, polymer processing industry

Principal current tasks:

- Testing fluorescent star polymers as probes

- Developing a series of fluorescent stars with well-defined sizes and independent emission spectra

- Observing the behavior of the stars in immiscible polymer blends using advanced optical microscopy techniques

- Determine whether the stars migrate to phase boundaries to help explain how highly branched polymers affect the properties of immiscible blends.

\section{Additional information:}

Fluorescent sub-micron probe molecules are needed to characterize structures and transport properties of membranes, polymer blends, and biomaterials. An ideal fluorescent probe would exhibit monodisperse size, versatile solubility, high luminescence, minimal photobleaching, and stability under conditions of varying temperature, solvent, and $\mathrm{pH}$. Taken together, these criteria are rather demanding, making the development of model probes a challenge.

\section{Selected publications:}

Hedden, R.; Amis, E.J.; Gröhn, F; Bauer, B.J; "Novel Fluorescent 'Probe' Molecules: Dendrimer-Star Polymers Containing Cadmium Sulfide Nanoparticles", submitted to Macromolecules.

Alignment with NSET Grand Challenges:

\begin{tabular}{|c|c|c|c|c|c|c|c|c|c|c|}
\hline MAT & ELEC & HEALTH & ENV & ENER & SPACE & BIOSEN & TRANS & SECUR & MET & MAN \\
\hline $\mathrm{X}$ & $\mathrm{X}$ & & & & & & & & $\mathrm{X}$ & \\
\hline
\end{tabular}




\section{Materials Science and Engineering Laboratory}

\section{Fundamental Studies for the Development of Photoresists for Next-generation Photolithography: Thin Film Chain Conformation and Polymer Dynamics}

\section{Project Leader/contact:}

Eric Lin, 301-975-6743, eric.lin@ nist.gov

Polymers Division, MSEL, Gaithersburg

Approximate staffing:

6.4 FTE

Funding sources:

NIST (60\%), Other Agencies (30\%), Other (10\%)

Objective:

Establish a new methodology to measure quantitatively line edge and sidewall roughness of lithographically produced patterns with deep sub-micrometer feature sizes. Develop and apply high spatial resolution measurements to obtain unique data needed to enable the development of next-generation photoresists. Determine relative contribution of material and interfacial factors in photoresist and anti-reflective coating formulations on profile control, critical dimension, and roughness.

\section{Constituency:}

Semiconductor electronics industry and nanoscale manufacturers

Principal current tasks:

- Small-angle neutron scattering (SANS) measurements of chain conformation of model photoresist polymers

- Benchmark SANS metrology of critical dimension and roughness of patterned structures

- Identify contributing factors to photoresist performance on bottom anti-reflective coatings (BARCs)

- Complete measurement of deprotection reaction with standing wave exposure

- Determine feasibility of modulus measurement of patterned structures

Additional information:

Photolithography remains the driving technology in the semiconductor industry to fabricate integrated circuits with ever decreasing feature sizes. Today, most fabrication facilities use chemically-amplified (CA) photoresists, complex and highly tuned formulations of a polymer film loaded with photoacid generators (PAGs) and other additives. Upon exposure of the photoresist film through a mask, the PAG releases acidic protons. A post-exposure bake is then applied and the acid protons diffuse and catalyze a deprotection reaction on the polymer that alters its solubility in an aqueous base developer solution. These reactive and diffusive processes must be understood and controlled at the nanometer length scale to effectively fabricate integrated circuits.

\section{Selected publications:}

Wu, W.L., Lin, E.K., Lin, Q.H., Angelopoulos, M. "Small Angle Neutron Scattering Measurements of

Nanoscale Lithographic Features," J. Appl. Phys. 88, 7298 (2000).

Alignment with NSET Grand Challenges:

\begin{tabular}{|c|c|l|l|l|l|l|l|c|c|c|}
\hline MAT & ELEC & HEALTH & ENV & ENER & SPACE & BIOSEN & TRANS & SECUR & MET & MAN \\
\hline $\mathrm{X}$ & $\mathrm{X}$ & & & & & & & & $\mathrm{X}$ & $\mathrm{X}$ \\
\hline
\end{tabular}




\section{Materials Science and Engineering Laboratory}

\section{Combinatorial Methods for Polymer Nanocomposite Film Properties}

\section{Project Leader/contact:}

Alamgir Karim, 301-975-6588, alamgir.karim@nist.gov

Polymers Division, MSEL, Gaithersburg

Approximate staffing:

1.2 FTE

Funding sources:

NIST (70 \%), Other Agency (30\%)

Objective:

Develop combinatorial techniques to fabricate and characterize phase behavior of nanocomposite polymer blend films.

Develop combinatorial methods to measure barrier properties of nanocomposite polymer films.

Constituency:

Automotive industry, nanoscale sensors and instrumentation, nanocomposite compounders

Principal current tasks:

- Phase behavior determination

- Morphology mapping

- Gas and vapor barrier properties measurements

\section{Additional information:}

Polymer materials are often mixed with inorganic materials in the bulk to enhance properties including mechanical, electrical, thermal, and physical. Such property enhancements are induced not only by the physical presence of the filler but also significantly by the interaction of the polymer with the filler via altering the local properties of the polymer material. In this regard, recently layered silicate nanocomposites have been shown to be effective in modifying the polymer properties due to their high surface area of contact between the polymer and the high aspect ratio nanoparticle. Potential property enhancements should also occur in polymer nanocomposite thin films due to nanoparticle orientation from film confinement effects. We investigate the effect of layered silicate nanoparticles on the phase behavior of a classic polymer blend system using high throughput combinatorial methods. Methods to combinatorially measure oxygen and water vapor barrier properties are also being developed.

Selected publications:

1. A. Karim*, K.Yurekli, C. Meredith, and E.J. Amis, "Combinatorial Methods for Polymer Materials Science: Phase Behavior of Nanocomposite Blend Films", Polymer Engineering \& Science, In Press.

2. V. Ferreiro, G. Schmidt, C.C. Han, A. Karim*, "Dispersion and Nucleating Effects of Clay Fillers in Nanocomposite Polymer Films", American Chemical Society, Symp. Proc., Vol 804, 2001.

3. K.A. Barnes, A. Karim*, J.F. Douglas, A.I. Nakatani, H. Gruell, E.J. Amis, "Suppression of Dewetting in Nanoparticle-Filled Polymer Films", Macromolecules, 33, 4177 (2000).

Alignment with NSET Grand Challenges:

\begin{tabular}{|l|l|l|l|l|l|l|l|l|l|l|}
\hline MAT & ELEC & HEALTH & ENV & ENER & SPACE & BIOSEN & TRANS & SECUR & MET & MAN \\
\hline $\mathrm{X}$ & & & & & & $\mathrm{X}$ & & & $\mathrm{X}$ & \\
\hline
\end{tabular}




\section{Materials Science and Engineering Laboratory}

\section{Polymer Nanocomposites}

\section{Project Leader/contact:}

Dr. Erik Hobbie, 301-975-6774, erik.hobbie@ nist.gov

Polymers Division, MSEL, Gaithersburg

Approximate staffing:

5.1 FTE

Funding sources:

NIST (100\%)

Objective:

Develop characterization methods, including microstructure measurements and modeling tools, to connect the structure and alignment of nanocomposites consisting of polymers and nanofillers with the processing conditions, and relate this microstructure to bulk mechanical and dielectric properties.

Constituency:

Automotive parts industry, polyolefin materials producers, nanocomposites industry

Principal current tasks:

- Quantify dispersion, alignment, and structure via small angle scattering and electron microscopy

- Establish on-line metrology for bulk dielectric and mechanical measurements

- Establish a theoretical and empirical quantitative link between nano-structure and bulk properties that points toward efficient in-line "bulk" measurements of nano-structure.

- Establish methods for relating the conditions measured and controlled during material processing to the final properties of interest for nanocomposites.

\section{Additional information:}

Inorganic fillers frequently are used to enhance polymer properties. New attributes may be achieved with nanoparticulates provided that the underlying science is developed. Polymer nanocomposites are important in many diverse industrial processes. Potential applications derived from synergistic behaviors and hybrid properties of polymer-nanoparticle composites include data storage and optical and electro-rheological materials for display devices.

\section{Selected publications:}

1. M.M. McBrearty, A. J. Bur, S.C. Roth, "Variation of Electrical Properties with Exfoliation Condition in Nanocomposites" ANTEC 2002

2. G. Schmidt, A. I. Nakatani, P. D. Butler, and C. C. Han, "Shear Orientation of Viscoelastic Polymer-Clay Solutions Probed by Flow Birefringence and SANS", Macromolecules, 33, 7219 (2000).

3. C. C. Han, G. Schmidt, A. I. Nakatani, and P. Butler, "A Small Angle Neutron Scattering Study on Polymer Clay Solutions”, ACS PMSE, 포, 219, Chicago, Sept. (2001).

Alignment with NSET Grand Challenges:

\begin{tabular}{|c|c|c|c|c|c|c|c|c|c|c|}
\hline MAT & ELEC & HEALTH & ENV & ENER & SPACE & BIOSEN & TRANS & SECUR & MET & MAN \\
\hline $\mathrm{X}$ & & & & & & & $X$ & & $X$ & $X$ \\
\hline
\end{tabular}




\section{Materials Science and Engineering Laboratory}

\section{Giant Magnetoresistance Materials}

\section{Project Leader/contact:}

William F. Egelhoff, Jr., 301-975- 2542, william.egelhoff@nist.gov

Metallurgy Division, MSEL, Gaithersburg

Approximate staffing:

5.0 FTE

Funding sources:

NIST ( $88 \%)$, Other Agencies (12\%)

\section{Objective:}

Synthesize and characterize new magnetoresistive materials and structures of reduced dimensionality through the precise control provided by electrochemical and physical vapor deposition.

\section{Constituency:}

The ultrahigh density data storage industry, which needs improved magnetoresistive materials for use in harddisk drives and in magnetoresistive random access memory chips (IBM, Motorola, Honeywell, Seagate,

Maxtor, etc.)

Principal current tasks:

- Optimizing the fabrication of giant magnetoresistive (GMR) materials and magnetoresistive nanocontacts.

Additional information:

GMR materials are already widely used in computer hard-disk drives, and emerging markets include nonvolatile memory chips, magnetic field sensors, and ultrahigh speed isolators. However, continued progress requires improved magnetoresistive materials. We help these companies learn how to produce improved GMR materials, and we are investigating novel advanced concepts in magnetoresistive nanocontacts that may someday replace GMR materials. Our work provides U.S. companies with significant competitive help by investigating the science underlying the manufacturing process. Advances in the field may make possible new generations of small, rugged, inexpensive magnetic sensors for a wide range of applications as diverse as position sensing in automated manufacturing and magneto-encephlography. As a result, within a few years we are likely to be surrounded in our daily lives by products that make use of new magnetoresistive materials.

Selected publications:

"Oxygen as a Surfactant in the Growth of GMR Spin Valves," W. F. Egelhoff, Jr., P. J. Chen, C. J. Powell, M.

D. Stiles, R. D. McMichael, J. H. Judy, K. Takano,and A. E. Berkowitz, J. Appl. Phys., 82, 6142 (1997)

Alignment with NSET Grand Challenges:

\begin{tabular}{|c|c|c|c|c|c|c|c|c|c|c|}
\hline MAT & ELEC & HEALTH & ENV & ENER & SPACE & BIOSEN & TRANS & SECUR & MET & MAN \\
\hline $\mathrm{X}$ & $\mathrm{X}$ & & & & & & & & $\mathrm{X}$ & $\mathrm{X}$ \\
\hline
\end{tabular}




\section{Materials Science and Engineering Laboratory}

\section{Magnetic Properties of Nanostructured Materials}

\section{Project Leader/contact:}

Robert D. Shull, 301-975-6035, robert.shull@nist.gov

Metallurgy Division, MSEL, Gaithersburg

Approximate staffing:

2.0 FTE

Funding sources:

NIST (75\%), Other Agencies (25\%)

\section{Objective:}

This project is directed toward understanding the magnetic character of materials possessing some critical dimension at the nanometer level.

Constituency:

Magnetic data recording industry, theft control and health industries, refrigeration industry, and remotelycontrolled smart-materials industry.

Principal current tasks:

- To measure the magnetocaloric effects in dysprosium- and holmium-substituted magnetic garnet nanocomposites containing $\mathrm{Fe}, \mathrm{Co}$, and $\mathrm{Ni}$

- To measure the magnetic properties of different composition $\mathrm{C}_{60} / \mathrm{Fe}$ and $\mathrm{C}_{60} / \mathrm{Ni}$ thin films.

- To measure the magnetic characteristics of nanometer-sized $\mathrm{Fe}-$, $\mathrm{Ni}$-, and $\mathrm{Co}-$ encapsulated particles in polyamidoamine (PAMAM) dendrimer hosts, embedded in a polystyrene matrix.

- To measure the composition and dilution dependence of magnetic nanoparticles in carboxylic acid, water, and alkylnaphthalene.

\section{Additional information:}

Magnetic nanocomposites possess characteristic length scales comparable to critical magnetic lengths, and therefore may be expected to possess much different magnetic character than conventional large-length scale materials. Improvements in efficiency, magnetic capabilities, and property combinations are expected. In order for industry to utilize these materials in magnetic recording, cooling, drug delivery, and nano-electro-mechanical systems (NEMS), their character needs to be understood. We have 15 years of expertise in the area of magnetic nanocomposites.

Selected publications:

1. "Formation of Nanomagnetic Thin Films by Dispersed Fullerenes," L.A. Zheng, B.M. Lairson, E.V. Barrera, and R.D. Shull, Appl. Physics Letters 77, No. 20, 3242 (2000).

2. "Magnetocaloric Effect of Ferromagnetic Particles," R.D. Shull, IEEE Trans. on Magnetics 29, No. 6, 2614 (1993).

Alignment with NSET Grand Challenges:

\begin{tabular}{|c|c|c|l|c|l|l|l|c|c|c|}
\hline MAT & ELEC & HEALTH & ENV & ENER & SPACE & BIOSEN & TRANS & SECUR & MET & MAN \\
\hline $\mathrm{X}$ & $\mathrm{X}$ & $\mathrm{X}$ & & $\mathrm{X}$ & & & & & $\mathrm{X}$ & \\
\hline
\end{tabular}




\section{Materials Science and Engineering Laboratory}

\section{Magnetic Domain Motion in Nanostructured Materials}

\section{Project Leader/contact:}

Robert D. Shull, 301-975-6035, robert.shull@nist.gov

Metallurgy Division, MSEL, Gaithersburg

Approximate staffing:

2.25 FTE

Funding Sources:

NIST $(100 \%)$

Objective:

To demonstrate the utility of an inexpensive magneto-optical tool for observing magnetic domains, and their dynamics, in nanostructured materials in order to determine the mechanism whereby a material reverses its magnetization vector upon the application of a reversed external magnetic field. Such information is crucial to understanding the material's magnetic hysteresis loop, a property that controls application of the material.

Constituency:

Magnetic recording industry, magnetic sensor companies, permanent magnet manufacturers

Principal current tasks:

- Observation of the remagnetization in an $\mathrm{FeMn} / \mathrm{NiFe}$ antiferromagnet/ferromagnet exchange-biased couple ac-demagnetized at high temperature in order to observe domain effects in the antiferromagnet.

- Observation of the remagnetization mechanism in a $\mathrm{SmCo} / \mathrm{Fe}$ hard/soft ferromagnet couple in order to verify a coherent rotation model proposed 10 years ago, but as yet unverified.

- Observation of the effects of pinholes in the nanometer-thick Ru layer separating two NiFe ferromagnetic layers, a multiplayer structure now being proposed by IBM for the next generation recording media.

\section{Additional Information:}

Observation of the dynamics of magnetic domains is very difficult, and few techniques allow one to do it. The Magneto-Optical Indicator Film (MOIF) technique is an inexpensive method for studying domain dynamics that was developed by researchers from the Russian Academy of Sciences and NIST. It is well suited for investigating the new nanostructured magnetic materials being developed for numerous applications, including magnetic recording media and sensors.

\section{Selected Publications:}

1. "Spin Spring Behavior in Exchange Coupled Soft and High-Coercivity Hard Ferromagnets," R.D. Shull, A.J. Shapiro, V.S. Gornakov, V.I. Nikitenko, J. S. Jiang, H. Kaper, G. Leaf, and S.D. Bader, IEEE Transactions on Magnetics 37, No. 4, 2576 (2001).

2. “Anisotropy-Dependent Macroscopic Domain Structure in Wedged-Permalloy/Uniform-FeMn Bilayers," Kai Liu, S.M. Zhou, C.L. Chien, V.I. Nikitenko, V.S. Gornakov, A.J. Shapiro, and R.D. Shull, J. Appl. Phys. 87 (9), 5052 (2000).

Alignment with NSET Grand Challenges:

\begin{tabular}{|l|l|l|l|l|l|l|l|l|l|l|}
\hline MAT & ELEC & HEALTH & ENV & ENER & SPACE & BIOSEN & TRANS & SECUR & MET & MAN \\
\hline $\mathrm{X}$ & $\mathrm{X}$ & & & & & & & & $\mathrm{X}$ & \\
\hline
\end{tabular}




\section{Materials Science and Engineering Laboratory}

\section{Nanoscale Magnetic SRMs and Characterization Techniques}

\section{Project Leader/contact:}

- Robert D. Shull, 301-975-6035, robert.shull@ nist.gov

Metallurgy Division, MSEL, Gaithersburg

- David P. Pappas, 303-497-3374, pappas@boulder.nist.gov

Magnetic Technology Division, EEEL, Boulder

Approximate staffing:

2.0 FTE

Funding Sources:

NIST (100\%)

Objective:

This project involves measurement and characterization of nanoscale magnetic materials. This activity includes the preparation of standard reference materials, where appropriate, in order to accelerate the development and application of this class of advanced materials.

Constituency:

Magnetic recording industry, magnetic sensor companies, permanent magnet manufacturers, magnetic measuring equipment manufacturers

Principal current tasks:

- Investigating feasibility of making a thin film magnetic standard.

- Evaluation of a thin film superconducting coil magnetic inductance standard.

- Measurement of magnetic aftereffect and time dependence in nanoparticulate $\mathrm{Fe}$ on tape magnetic recording media.

- Preisach modeling of hysteresis loops of nanoparticulate recording media.

Additional Information:

Magnetic materials are widely used in transformers, motors, and recording devices. This group has 15 years of expertise in the measurement and characterization of nanoscale magnetic materials, and has developed and constructed a novel magnetometer to measure a material's "Absolute Magnetic Moment." In addition, this group has an expertise in correlating material structure and properties.

\section{Selected Publications:}

“Absolute Magnetic Moment Measurements of Nickel Spheres,” R.D. Shull, R.D. McMichael, L.J.

Swartzendruber, and S.D. Leigh, J. Appl. Phys. 87, No. 9, 5992 (2000).

Alignment with NSET Grand Challenges:

\begin{tabular}{|c|c|c|c|c|c|c|c|c|c|c|}
\hline MAT & ELEC & HEALTH & ENV & ENER & SPACE & BIOSEN & TRANS & SECUR & MET & MAN \\
\hline $\mathrm{X}$ & $\mathrm{X}$ & & & & & & & & $\mathrm{X}$ & \\
\hline
\end{tabular}




\section{Materials Science and Engineering Laboratory}

\section{Magnetic Dynamics and Micromagnetics of Thin Film}

\section{Project Leader/contact:}

Robert D. McMichael, 301-975-5121, robert.mcmichael@nist.gov

Metallurgy Division, MSEL, Gaithersburg

Approximate staffing:

2.0 FTE

Funding sources:

NIST $(100 \%)$

Objective:

To provide models of magnetization damping, dynamic measurement methods, materials data, and micromagnetic modeling tools and benchmarks

Constituency:

Magnetic data storage industry

Principal current tasks:

0 To identify and describe the physical mechanisms that govern magnetization damping and to develop ways to measure and control them.

\section{Additional information:}

For many device applications of magnetic thin films, it is becoming increasingly important to control the magnetization on sub-micron and sub-nanosecond time scales. The damping properties of the magnetization are especially important for high data rate applications, and the dynamic behavior of submicron magnetic particles are important for high density data storage. The damping mechanisms that couple the magnetization to the thermal bath also cause magnetization fluctuations that are becoming an important source of noise in next-generation read heads and a mechanism for data loss in media.

Selected publications:

1. http://www.ctcms.nist.gov/ rdm/mumag.html.

2. R. D. McMichael, M. J. Donahue, D. G. Porter and J. Eicke, "Switching dynamics and critical behavior of standard problem No. 4," J. Appl. Phys., 89, 7603 (2001).

3. R. D. McMichael, M. D. Stiles, P. J. Chen and W. F. Egelhoff, Jr., "Ferromagnetic resonance linewidth in thin films coupled to NiO” J. Appl. Phys., 83, 7037 (1998).

4. R. D. McMichael and Andrew Kunz, "Calculation of damping rates in thin inhomogeneous ferromagnetic films due to coupling to lattice vibrations," J. Appl. Phys., to be published (2002).

5. R. D. McMichael, D. J. Twisselmann, J. E. Bonevich, P. J. Chen, W. F. Egelhoff, Jr., and S. E. Russek, "Ferromagnetic resonance mode interactions in periodically perturbed films," J. Appl. Phys., to be published (2002).

Alignment with NSET Grand Challenges:

\begin{tabular}{|c|c|c|l|l|l|l|l|c|c|c|}
\hline MAT & ELEC & HEALTH & ENV & ENER & SPACE & BIOSEN & TRANS & SECUR & MET & MAN \\
\hline $\mathrm{X}$ & $\mathrm{X}$ & & & & & & & & $\mathrm{X}$ & \\
\hline
\end{tabular}




\section{Materials Science and Engineering Laboratory}

\section{Neutron Methods for Nanotechnology}

\section{Project Leader/contact:}

- John J. Rush, 301-975-6231, john.rush@ nist.gov

- Patrick Gallagher, 301-975-4191, patrick.gallagher@ nist.gov

NIST Center for Neutron Research (NCNR), NIST, Gaithersburg

Approximate staffing:

6.0 FTE

Funding sources:

NIST (67\%), Other Agencies (33\%)

\section{Objective:}

Provide unique national capabilities and instrumentation for neutron research on nanoscale structure $(1 \mathrm{~nm}-100 \mathrm{~nm})$ and dynamics, which control properties and nanotechnology applications of materials and macromolecules.

Constituency:

Over 40 of U.S. universities and industries and three NIST laboratories pursuing nanoscale science and technology research at NCNR

\section{Principal current tasks}

- Over 100 NIST and outside U.S. researchers are engaged in research aimed at probing nanostructure in new materials and assemblages which are key to new development, e.g. in magnetic storage media and spin electronics, nanocomposites for automotive ports, thin film insulators for nanoscale integrated circuits, biomimetic membranes for biosensor applications, tailored molecular structures for molecular sieves, catalysts and fuel additives and nanoscopic molecular assemblies for drug delivery.

- NCNR is working to develop new methods and devices to improve sensitivity of neutron probes for nanostructure measurement by an order of magnitude.

\section{Additional information:}

Developments at the NCNR over the past decade provide U.S. nanoscale science and technology with timely access to state-of-the-art neutron research capabilities. More opportunities to meet rapidly emerging nanomeasurement opportunities are being explored.

\section{Selected publications:}

Over 50 publications in last 2 years

Alignment with NSET Grand Challenges:

\begin{tabular}{|c|c|c|c|c|c|c|c|c|c|c|}
\hline MAT & ELEC & HEALTH & ENV & ENER & SPACE & BIOSEN & TRANS & SECUR & MET & MAN \\
\hline $\mathrm{X}$ & $\mathrm{X}$ & $\mathrm{X}$ & $\mathrm{X}$ & $\mathrm{X}$ & & $\mathrm{X}$ & $\mathrm{X}$ & & $\mathrm{X}$ & \\
\hline
\end{tabular}




\section{Materials Science and Engineering Laboratory}

\section{Neutron Studies of Nanoscale Films and Multilayers}

\section{Project Leader/contact:}

Chuck Majkrzak, 301-975-5251, charles.majkrzak@nist.gov

NIST Center for Neutron Research (NCNR), NIST, Gaithersburg

Approximate staffing:

5.0 FTE

Funding sources:

NIST (90\%), Other Agencies (10\%)

Objective:

Measure key nanoscale magnetic and macromolecular structures in thin films and multilayers to support their tailoring and development, e.g. for advanced magnetic storage, microelectronic, and biosensor applications.

Constituency:

Communications, electronics, and biotechnology industries, and universities

Principal current tasks

- Extend magnetic thin films studies to next generation magnetic semiconductors to reveal the role of magnetic interactions, which will control the spin dependent properties of future spintronic devices.

- Measurement of polymer interdiffusion aggregation and growth of metal nanoparticles in thin films for microelectronic and coating applications.

- Develop theoretical and experimental methods to provide direct inversion of phase sensitive reflectivity measurements for accurate nanoscale structure determination.

\section{Additional information:}

NCNR scientists have developed powerful neutron reflectometry probes that provide essential nanoscale structure information that cannot be measured any other way.

\section{Selected publications:}

1. H. Yim, M. Kent, R. Ivkov, S. Satija, J. Majewski, "Structure Within Thin Epoxy Films Revealed by Solvent Swelling: A Neutron Reflectivity Study", Macromolecules 34, 7932 (2000).

2. C. F. Majkrzak, N. F. Berk, S. Krueger, J. A. Dura, M. Tarek, D. Tobias, V. Silin, C. W. Meuse, J. Woodward, A. L. Plant, "First-Principles Determination of Hybrid Bilayer Membrane Structure by Phase-Sensitive Neutron Reflectometry", Biophys. J. 79, 3330 (2000).

3. Borchers, J. A., Ijiri, Y., Lind, D. M., Ivanov, P. G., Erwin, R. W., Qasba, A., Lee, S.-H., O’Donovan, K. V., Dender, D. C., "Detection of Field-Dependent Antiferromagnetic Domains in Exchange-Biased $\mathrm{Fe}_{3} \mathrm{O}_{4} / \mathrm{NiO}$ Superlattices", Appl. Phys. Lett. 77 (25), 4187 (2000).

Alignment with NSET Grand Challenges:

\begin{tabular}{|c|c|c|l|l|l|l|l|c|c|c|}
\hline MAT & ELEC & HEALTH & ENV & ENER & SPACE & BIOSEN & TRANS & SECUR & MET & MAN \\
\hline $\mathrm{X}$ & $\mathrm{X}$ & & & & & $\mathrm{X}$ & & & $\mathrm{X}$ & \\
\hline
\end{tabular}




\section{Materials Science and Engineering Laboratory}

\section{Neutron Scattering Investigations of Nanocomposites, Nanotubes and Self Assembled Structures}

\section{Project Leader/contact:}

John J. Rush, 301-975-6231, john.rush@nist.gov

NIST Center for Neutron Research (NCNR), NIST, Gaithersburg

Approximate staffing:

$2.5 \mathrm{FTE}$

Funding sources:

NIST $(100 \%)$

Objective:

Provide critical information by small-angle neutron scattering (SANS) and diffraction methods on nanostructures in bulk nanocomposites for next generation structural materials, carbon nanotubes for molecular electronics, and new self-assembled macromolecules structures for environmental cleanup. First principles computational methods are a key to progress in predicting and tailoring nanotube properties.

\section{Constituency:}

Chemical, transportation and microelectronics sectors

\section{Principal current tasks}

- Use ultra-high resolution small-angle neutron scattering (USANS) to verify and calibrate the relationship between the color of organically modified clays dispersed in polymer matrices and the degree of exfoliation of the clay particles, which is strongly correlated with improved mechanical and fire retardant properties.

- Combine transmission electron microscopy (TEM), SANS, and dynamic rheology to develop a detailed structural and mechanical model of a polymerized gel. Characterizing the properties of this new micellar gel material will foster its commercial applications, e.g. in wastewater cleanup.

- Explore new routes to synthesize and characterize functionalized derivatives of carbon nanotubes, such as hydrogen and metal-coated tubes.

Additional information:

The NIST/NCNR small angle scattering instruments cover a range from $1 \mathrm{~nm}$ to $1000 \mathrm{~nm}$ and thus can uniquely probe many key features in molecular and particulate nanostructures.

\section{Selected publications:}

1. T. Yildirim, O. Gulseren, C. Kilic, S. Ciraci, "Pressure-Induced Interlinking of Carbon Nanotubes", Phys. Rev. $B$ 62, 12648 (2000).

2. D. L. Ho, R. M. Briber, C. J. Glinka, "Characterization of Organically Modified Clays Using Scattering and Microscopy Techniques", Chem. Mater. 13 (5), 1923 (2001).

3. S. R. Kline, "Structural Evolution During Micelle Polymerization”, J. Appl. Crystallogr. 33 (1), 618 (2000).

Alignment with NSET Grand Challenges:

\begin{tabular}{|c|c|c|c|c|c|c|c|c|c|c|}
\hline MAT & ELEC & HEALTH & ENV & ENER & SPACE & BIOSEN & TRANS & SECUR & MET & MAN \\
\hline $\mathrm{X}$ & $\mathrm{X}$ & & $\mathrm{X}$ & & & & $\mathrm{X}$ & & $\mathrm{X}$ & \\
\hline
\end{tabular}




\section{Building and Fire Research Laboratory}

\section{Characterization of Polymer Surfaces}

\section{Project Leader/contact:}

Mark VanLandingham, 301-975-4686, mark.vanlandingham@nist.gov

Building Materials Division, BFRL, Gaithersburg

Approximate staffing:

1.2 FTE

Funding sources:

NIST (92\%), Other (8\%)

Objective:

To develop advanced measurement techniques for evaluating surface mechanical properties of polymeric materials as a function of time and loading rate, to relate materials properties to deformation behavior under complex stress states, and to assess the impact of surface deformation on appearance.

Constituency:

This project addresses issues of micromechanical and nanomechanical behavior of polymers that are applicable to a number of industries, including building and construction, automotive, bulk plastics, and microelectronics.

\section{Principal current tasks}

- Develop techniques using nanoindentation to measure elastic, viscoelastic, and viscoplastic responses of amorphous and semicrystalline homopolymer systems.

\section{Additional information:}

This project has become the BFRL-led portion of the Interfaces/Interphases Consortium that was established in December 2000. In the Consortium, BFRL partners with MSEL and CSTL, and three private companies. Results have shown the feasibility of mechanical property measurements on polymeric materials using atomic force microscopy and depth-sensing instrumentation. Information has also been obtained on the effects of probe tip shape and its importance to the quantitative determination of mechanical properties from nanoscale indentation measurements. Models of optical scattering have been developed for clear coatings with varying surface topography that correlate well with experimental measurements. A review of the literature on marring of surfaces has been completed, but publication is delayed for six months in accord with requirements of the Consortium.

\section{Selected publications:}

M. R. VanLandingham, J. S. Villarrubia, W. F. Guthrie, G. F. Meyers, "Nanoindentation of Polymers: An

Overview," in Macromolecular Symposia 167: Advances in Scanning Probe Microscopy of Polymers, V. V.

Tsukruk and N. D. Spencer, eds. (2001) 15-44.

Alignment with NSET Grand Challenges

\begin{tabular}{|l|l|l|l|l|l|l|l|c|c|c|}
\hline MAT & ELEC & HEALTH & ENV & ENER & SPACE & BIOSEN & TRANS & SECUR & MET & MAN \\
\hline & & & & & & & & & $X$ & \\
\hline
\end{tabular}




\section{Building and Fire Research Laboratory}

\section{High-Performance Polymeric Building Materials: Polymer-Metal Oxide Nanocomposite Systems}

Project Leader/contact:

Jonathan Martin, 301-975-6717, jonathan.martin@nist.gov

Building Materials Division, BFRL, Gaithersburg

Approximate staffing:

3.8 FTE

Funding sources:

NIST (68\%), Other Agencies (32\%)

Objective:

To develop measurement technology related to the use of metal oxide nanoparticles $\left(\mathrm{TiO}_{2}, \mathrm{ZnO}\right)$ in polymeric building materials; to develop dispersion measurement methods using a combination of light and neutron scattering metrologies; to investigate relationships between dispersion and bulk and nanoscale mechanical and optical properties; to develop methods to measure properties related to the photoreactivity of the nanoparticles.

Constituency:

This project addresses issues relevant to building and construction materials, automotive coatings, and bulk plastics, with potential impacts to the healthcare industry.

Principal current tasks

- Investigate techniques to disperse pigmentary and nanosize particles in polymeric binders; develop and evaluate scattering methods for quantitative characterization of dispersion.

Additional information:

This is a new research project as of fiscal year 2002.

Alignment with NSET Grand Challenges

\begin{tabular}{|c|c|c|c|c|c|c|c|c|c|c|}
\hline MAT & ELEC & HEALTH & ENV & ENER & SPACE & BIOSEN & TRANS & SECUR & MET & MAN \\
\hline $\mathrm{X}$ & & $\mathrm{X}$ & & & & & & & $\mathrm{X}$ & \\
\hline
\end{tabular}




\section{Building and Fire Research Laboratory}

\section{Measurement Science for Optical Reflectance Scattering}

\section{Project Leader/contact:}

Mary McKnight, 301-975-6714, mary.mcknight@ nist.gov

Building Materials Division, BFRL, Gaithersburg

Approximate staffing:

0.7 FTE

Funding sources:

NIST $(100 \%)$

Objective:

To develop improved measurement methods and models for describing and predicting the optical reflectance and light scattering of materials.

Constituency:

Automotive, coatings, plastics, building and construction, and computer graphics industries.

Principal current tasks

- Complete characterization of the microstructure of metallic-flaked coatings jointly with MEL

- Develop light scattering laboratory for characterizing microstructure of clear and pigmented coatings

- Accurately render the appearance of objects coated with a metallic-flake paint.

Additional information:

The main contribution to nanotechnology has been the recently begun use of the NIST reactor to study the degree of dispersion of submicrometer particles in coatings.

\section{Selected publications:}

L. Sung, M. E. Nadal, M. E. McKnight, E. Marx, and B. Laurenti, "Optical Reflectance of Metallic Coatings: Effect of Aluminum Flake Orientation," submitted to JCT: Journal of Coatings Technology (2001).

Alignment with NSET Grand Challenges:

\begin{tabular}{|c|l|l|l|l|l|l|c|c|c|c|}
\hline MAT & ELEC & HEALTH & ENV & ENER & SPACE & BIOSEN & TRANS & SECUR & MET & MAN \\
\hline $\mathrm{X}$ & & & & & & & $\mathrm{X}$ & & $\mathrm{X}$ & \\
\hline
\end{tabular}




\section{Building and Fire Research Laboratory}

\section{New Flame Retardant Principles: \\ Condensed Phase Processes \\ Demonstration and Products Transfer \\ Pre-Flaming Behavior of Solid Materials}

Project Leader/contact:

Jeff Gilman, 301-975-6573, jeffrey.gilman@ nist.gov

Fire Research Division, BFRL, Gaithersburg

Approximate staffing:

6.5 FTE

Funding sources:

NIST (70 \%), Other Agencies (20\%), Other (10\%)

Objective:

To reduce the flammability of commodity polymers while simultaneously improving their physical properties.

Constituency:

Plastics and flame retardant product producers

Principal current tasks

- Develop several structure-property relationships for flame retardant polymer nanocomposites

- Develop high throughput (HT) methods to accelerate the pace of research in this program

Additional information:

In FY2001, work was completed within our NIST-Industry Nanocomposites Consortium in which the char forming properties of nano-clays in polystyrene were characterized. In cooperation with the Polymers Division, we also developed new quantitative NMR methods to characterize nano-scale mixing and processing degradation of nanocomposites. Working with the Naval Research Lab, we developed new clay treatments with greater thermal stability than other conventional treatments. As part of the NIST Combinatorial Methods Center, we are developing new high throughput methods for characterizing the flammability and physical properties of polymers.

Selected publications:

1. D. L. VanderHart, A. Asano, and J. W. Gilman, Macromolecules, 34, 3819, (2001).

2. J. W. Gilman, T. Kashiwagi, A. B. Morgan, R. Harris, Jr., L. Brassell, M. VanLandingham, and C. L. Jackson, NISTIR 6531, July 2000.

3. J. W. Gilman, T. Kashiwagi, "Polymer-Layered Silicate Nanocomposites with Conventional Flame Retardants" in Polymer-Clay Nanocomposites, eds.: T. Pinnavaia, G. Beall, Wiley and Sons Ltd, West Sussex, 2000.

Alignment with NSET Grand Challenges:

\begin{tabular}{|c|l|l|l|l|l|l|l|c|c|c|}
\hline MAT & ELEC & HEALTH & ENV & ENER & SPACE & BIOSEN & TRANS & SECUR & MET & MAN \\
\hline $\mathrm{X}$ & & & & & & & & & $\mathrm{X}$ & $\mathrm{X}$ \\
\hline
\end{tabular}




\section{Building and Fire Research Laboratory}

\section{Particle Measurements in Support of the Semiconductor Industry}

\section{Project Leader/contact:}

George Mulholland - 301-975-6695, george.mulholland@nist.gov

Fire Research Division, BFRL, Gaithersburg

Approximate staffing:

0.8 FTE

Funding sources:

NIST (92\%), Other (8\%)

Objective:

Develop facilities to accurately measure particle size and to deposit monosize particles on calibration artifacts to reduce the uncertainty in the sizes of particles used by the semiconductor industry to calibrate scanning surface inspection systems (SSIS).

Constituency:

Semiconductor electronics industry

Principal current tasks

Develop particle sizing and generation capabilities for particles as small as $10 \mathrm{~nm}$ using differential electrical mobility analysis for sizing and electrospray for aerosol generation.

Additional information:

The International Technology Roadmap for Semiconductors (ITRS) identifies the detection and characterization of defects and particles on wafers to be a potentially show-stopping barrier to device miniaturization. The roadmap specifies that by 2005 and $30 \mathrm{~nm}$ particles must be detectable on bare silicon. The manufacturers of the SSIS require low uncertainty calibration particles for use in calibrating surface scanners.

A major focus has been development of the differential mobility analysis (DMA) method for accurately sizing monosize polystyrene spheres. This work together with a SSIS round robin has provided evidence that current SSIS measurements have an unacceptably large uncertainty for particle sizes in the $90 \mathrm{~nm}$ to $100 \mathrm{~nm}$ size range. In addition, a spray pyrolysis method for generating pure copper spheres with diameters ranging from $100 \mathrm{~nm}$ to $200 \mathrm{~nm}$ has been developed in collaboration with the University of Minnesota. These spheres will be used to validate the particle scattering theory for particles on a silicon surface.

Selected Publications:

G. W. Mulholland and B. Bauer, "Nanometer Calibration Particles: What is Available and What is Needed?," J. Nanoparticle Research 2, 5-15 (2000).

Alignment with NSET Grand Challenges:

\begin{tabular}{|c|c|c|c|c|c|c|c|c|c|c|}
\hline MAT & ELEC & HEALTH & ENV & ENER & SPACE & BIOSEN & TRANS & SECUR & MET & MAN \\
\hline $\mathrm{X}$ & $\mathrm{X}$ & & & & & & & & $\mathrm{X}$ & \\
\hline
\end{tabular}




\section{Building and Fire Research Laboratory}

\section{A NIST Kinetic Data Base for PAH Reactions and Soot Particle Inception During Combustion}

\section{Project Leader/contact:}

George Mulholland - 301-975-6695, george.mulholland@nist.gov

Fire Research Division, BFRL, Gaithersburg

Approximate staffing:

3.5 FTE (including 0.25 from Sandia, 0.5 from AFRL, and 0.75 contractor)

Funding sources:

NIST (20\%), Other Agencies (80\%)

Objective:

To develop a critically evaluated, archival gas phase chemical kinetic database describing the transformation of fuel molecules to PAH (polycyclic aromatic hydrocarbons) and the first quantitative soot particle inception model based on experiments.

Constituency:

Gas turbine and diesel engine manufacturers

Principal current tasks

- Fabricate a well-stirred reactor, develop optical diagnostic for soot inception, assemble heptane pyrolysis and PAH growth kinetic databases, develop soot/PAH sampling methods, and measure PAH rates in shock tube.

Additional information:

Military gas turbine and diesel engines $=>$ emit soot and PAH. Engine particulate mitigation strategies need soot and PAH models for inclusion in computer-based engine design and for fuel additive development.

NIST is providing a new approach to understanding soot inception by focusing gas phase expertise and particle phase expertise on the nucleation of a condensed phase (particle) from large PAH molecules. The staff will be making use of new experimental facilities for detecting and sizing nanometer size particles, for measuring PAH/soot kinetics using improved well-stirred-reactor design with additive injection capabilities, and new computational tools for thermodynamic and kinetic parameters for combustion species including radicals and PAHs.

\section{Selected Publications:}

Blevins, L.G., Mulholland, G.W., Benner, B.A., Jr., Fletcher, R.A., and Steel, E.B., "Composition and Shape of Early Soot Collected from Inverse Flames," Fall Technical Meeting, Western States Section, The Combustion Institute, Salt Lake City, UT, October 2001.

Alignment with NSET Grand Challenges:

\begin{tabular}{|c|c|c|c|c|c|c|c|c|c|c|}
\hline MAT & ELEC & HEALTH & ENV & ENER & SPACE & BIOSEN & TRANS & SECUR & MET & MAN \\
\hline $\mathrm{X}$ & & $\mathrm{X}$ & $\mathrm{X}$ & $\mathrm{X}$ & & & $\mathrm{X}$ & & $\mathrm{X}$ & \\
\hline
\end{tabular}




\section{References}

[1]National Nanotechnology Initiative: Leading to the Next Industrial Revolution, NSTC Committee on Technology, Subcommittee on Nanoscale Science, Engineering and Technology, February 2000.

[2] Nanotechnology Research Directions: IWGN Workshop Report: Vision for Nanotechnology R\&D in the Next Decade, NSTC Committee on Technology, Interagency Working Group on Nanoscience, Engineering and Technology (IWGN), International Technology Research Institute, World Technology (WTEC) Division, Loyola College, September 1999.

[3] National Nanotechnology Initiative: The Initiative and Its Implementation Plan, NSTC Committee on Technology, Subcommittee on Nanoscale Science, Engineering and Technology, July 2000, pp. 19-20. [4] See the NNI website at http://www.nano.gov 